\title{
Pollution Claim Settlements under Correlated Information*
}

\author{
Martin Pesendorfer \\ Department of Economics, Yale University, New Haven, Connecticut 06520
}

Received February 23, 1994; revised October 17, 1997

\begin{abstract}
Agents have to decide whether a polluting plant will be operated or not. Agents' disutility (cost) from pollution is privately known, but correlated across agents. Incentive compatible mechanisms are characterized under the assumption that transfer payments are non-negative. The implementability constraint is similar to the independence case, except that virtual utilities are diminished by a factor due to the correlation of agents' types. As correlation vanishes, the implementability constraint approaches the implementability constraint of the independent types environment. It is established that the free-riding problem is relaxed as the number of agents increases. With many agents, ex post efficiency is achievable under ex ante budget balance. Journal of Economic Literature Classification Numbers: D82, H41.

(C) 1998 Academic Press
\end{abstract}

\section{INTRODUCTION}

It is well known that under the presence of external effects the market mechanism need not provide an efficient allocation. Ronald Coase suggested that this inefficiency can be alleviated by letting the involved parties bargain over the outcome. The "Coase Theorem" was that, if bargaining costs are negligible, side payments can be arranged such that the efficient outcome prevails. Recently, Rob [8] and Mailath and Postlewaite [5] proved this conjecture wrong: In large economies with independent private information there exists no bargaining mechanism that yields the efficient outcome; in fact, the probability of providing the efficient outcome is zero.

* This paper is based on a chapter of my thesis at Northwestern University (1995). I am grateful to V. V. Chari, Katerina Kyriazidou, Steven Matthews, Roger Myerson, Wolfgang Pesendorfer, Robert Porter, Asher Wolinsky, a seminar audience at Northwestern, two referees, and a co-editor of this journal for their helpful comments. I am especially grateful to one referee who provided very detailed and helpful comments. An Alfred P. Sloan Dissertation Fellowship and support from the Center of Urban Affairs and Policy Research is gratefully acknowledged. 
The reason is the strong bargaining power of individual agents. Only agents know their true valuations, and any incentive compatible mediation plan has to provide large enough side payments to low type agents to prevent them from imitating high type agents. In large economies, the additional incentive costs make it impossible for the mediator (government) to provide the project when it is socially efficient and balance the budget.

The analysis of Rob, and Mailath and Postlewaite relies on the assumption of independent private information. In many circumstances this does not seem appropriate. Consider "chloro-fluoro-carbons" emitting plants: Scientists findings that CFC emissions cause the shrinkage of the ozone layer, with negative effects on humans, are very likely to result in correlated opinions about these plants among economic agents. In general, if agents are allowed to communicate in the formation stage of private information, then private information may be correlated across agents. In this paper we suggest that mediation plans can make use of the fact that agents private valuations are correlated. The intuition is as follows: Each agent knows only his own valuation and infers the other agents' types conditional on his valuation. The mediator or government on the other hand, after having observed the announcements of $n-1$ agents, can infer the distribution of the valuation of the $n$th type conditional on the first $n-1$ valuations, and, since types are correlated, this is better than any single agent can do. Hence, in an incentive efficient mechanism the government knows more about the true distribution of the agents' valuations in the economy than any individual agent. Furthermore, this informational advantage of the government results in a weaker bargaining position of any single agent. It therefore seems plausible that the project will be provided more often than with independent valuations.

In fact, Myerson [7], Cremer and McLean [2, 3], McAfee and Reny [6] have shown in the context of auctions that, with correlated types, incentive constraints are not binding and hence the seller can extract the full surplus. The incentive efficient mediation plan exploits the differences in the conditional distributions of different types, by giving each type a lottery with possibly very large positive and negative payments.

When the correlation of types is small, these payments are very large. The problem with this mechanism is that it relies heavily on the assumption of risk neutral agents. This might be reasonable for example for oil companies bidding for drilling rights, but it does not seem appropriate for small economic agents who want compensation payments for pollution. One way to proceed is to impose risk aversion on the agents' utility. With this assumption the transfer payments have to be small in magnitude. Another way, and this we are going to pursue, is to restrict the allowable transfer payments, by imposing limited liability. We assume that the transfer payments to the agents have to be non-negative. 
We consider a simple model with discrete types of agents and characterize the set of incentive compatible mechanisms satisfying limited liability and ex ante budget balance. The implementability constraint looks similar to the independent type case except that the virtual costs are diminished by a factor due to the correlation of types. Since the virtual costs are lower, we can provide the project more often than with independent types. Robert [9] has shown, in the context of auctions, that, under limited liability, the optimal mechanism is continuous in the space of information distributions. Thus, by continuity, the independent types case can be viewed as a limiting case of the correlated information environment with limited liability. We establish that this result continues to hold in our setting.

We analyze the situation as the number of agents increases. We find that in large economies the project can be provided when it is socially efficient. In particular, the mechanism that maximizes the expected profits of the firm operating the polluting plant is efficient. Thus, the result of Rob, and Mailath and Postlewaite is not robust, in the sense that it can not be viewed as a limiting case of the correlated types environment with limited liability. The intuition is as follows. The optimal mechanism involves payments to high type agents only in the state where everybody is a high type agent. As the number of agents increases, low type agents will assign zero probability to the event that everybody else is a high type agent. Therefore, the virtual cost of low type agents decreases in the number of agents and vanishes in the limit.

Our analysis assumes ex ante budget balance. This has the advantage that our model is compatible with Rob, and Mailath and Postlewaite, but it has the disadvantage that the government, especially in the optimal mechanism, receives a lottery with possibly large positive and negative payments. It seems plausible that the transfer payments involved in a particular pollution claim settlement are small relative to the overall government budget, but it would still be desirable to reduce the variance in the government's lottery. An extreme way to do so is to impose ex post budget balance. We find that in large economies and under ex post budget balance the probability of provision is also positive.

The paper is organized as follows. Section 2 presents the model. Section 3 analyzes incentive compatible mechanisms under ex ante budget balance. Section 4 considers incentive compatible mechanisms under ex post budget balance. Conclusions are given in section 5 .

\section{MODEL}

Let $\mathscr{N}=\{1,2, \ldots, n\}$ be the set of economic agents. Let $s, t_{1}, t_{2}, \ldots, t_{n}$ be distinct random variables with the following properties: 
Assumption (i). The support of $s$ is $\mathscr{S}=[0,1]$, and the support of $t_{i}$ is $\mathscr{T}_{i}=\{0,1, \ldots, T\}$ for $i=1,2, \ldots, n .^{1}$

Assumption (ii). The random variable $s$ is drawn from a distribution with continuous density $f(s)$. The random variable $t_{i} \in \mathscr{T}_{i}$ is drawn from a distribution conditional on $s$ with conditional density $g\left(t_{i} \mid s\right)$ continuous in $s$. The density for $t_{i}$ is given by

$$
g\left(t_{i}\right)=\int_{\mathscr{S}} g\left(t_{i} \mid s\right) \cdot f(s) d s
$$

The joint density of $\left(s, t_{1}, t_{2}, \ldots, t_{n}\right)$ is given by

$$
h\left(s, t_{1}, t_{2}, \ldots, t_{n}\right)=g\left(t_{1} \mid s\right) \cdot g\left(t_{2} \mid s\right) \cdots g\left(t_{n} \mid s\right) \cdot f(s)
$$

We assume that conditional on $s$ the random variables $\left(t_{i}\right)_{i=1}^{n}$ are independent and identical distributed across consumers. Correlation between agents' types enters through the unobserved state variable $s .{ }^{2}$

Henceforth, we will denote by $\left(\hat{s}, \hat{t}_{1}, \hat{t}_{2}, \ldots, \hat{t}_{n}\right)=(\hat{s}, \hat{t})$ the realizations of the random variables $\left(s, t_{1}, t_{2}, \ldots, t_{n}\right)$ and by $t$ we will denote a generic element of $\mathscr{T}=\times_{i=1}^{n} \mathscr{T}_{i}$. We will occasionally use the notation $\left(t_{i}^{\prime}, t_{-i}\right)=$ $\left(t_{1}, t_{2}, \ldots, t_{i-1}, t_{i}^{\prime}, t_{i+1}, \ldots, t_{n}\right)$. With $E$ we denote the expectations operator with respect to the random variables $(t, s)$ and $E\left[., . \mid t_{i}\right]$ denotes the expectations operator with respect to $\left(t_{-i}, s\right)$ conditional on agent $i$ being of type $t_{i}{ }^{3}$

The problem that we are going to consider is whether to provide a project or not, for example whether to operate a polluting plant or not. If the plant is operated, the utility to agent $i$ is given by $x_{i}-t_{i}$, while if it is not operated the utility to agent $i$ is given by $x_{i}$, where $x_{i}$ is the transfer payment to agent $i(i=1,2, \ldots, n)$. The plant yields total revenues $R(n)$, or in per capita terms $r$, with $R(n)=r \cdot n$.

We study direct revelation mechanisms in which the probability of provision and the transfer payments are functions of the agents' reported

${ }^{1}$ With the term support of a probability measure we mean the following: A point $s$ is said to be in the support of a probability measure $\mu$ defined on $\mathscr{B}$ (the Borel sets) if and only if every open neighborhood of $s$ has strictly positive measure. The set of all such points is called the support of $\mu$.

${ }^{2}$ This formulation contains the independent types framework as a limiting case. To see this, consider a sequence of densities $f_{m}(s)$ that converge to the density of a one-point distribution. In the limit, as $m \rightarrow \infty$, the random variables $\left(t_{i}\right)_{i=1}^{n}$ are independently distributed.

${ }^{3}$ For any real valued function $G$ defined on $\mathscr{T}$, the expectations operator is given by $E[G]=\sum_{t \in \mathscr{T}} G(t) \int_{S} h(t, s) d s$ and the conditional expectations operator is given by

$$
E\left[G\left(t^{\prime}\right) \mid t_{i}\right]=\sum_{t_{-i}^{\prime} \in \mathscr{T}_{-i}} G\left(t_{-i}^{\prime}, t_{i}^{\prime}\right) \int_{S} \frac{h\left(t_{-i}^{\prime}, t_{i}, s\right)}{g\left(t_{i}\right)} d s .
$$


valuations. By the revelation principle, the restriction to direct revelation mechanism is without loss of generality, in the sense that any equilibrium outcome of some game will also be an equilibrium outcome of some revelation mechanism in which the agents report their types truthfully. A mechanism $\left(\left\langle x_{i}\right\rangle_{i=1}^{n}, q\right)$ consists of a collection of transfer payments $x_{i}(\hat{t}), x_{i}: \mathscr{T} \rightarrow \mathbf{R}$, and a probability function of providing the project $q(\hat{t})$, $q: \mathscr{T} \rightarrow[0,1]$. Since the conditional density $g(\cdot \mid s)$ is the same for all $i$, imposing anonymity on the mechanism ensures that agents with the same type are treated in the same way. A mechanism $(x, q)$ is anonymous if for any two agents $i, j$ with reports $\hat{t}_{i}=\hat{t}_{j}$ the transfer payments are equal, that is $x_{i}(\hat{t})=x_{j}(\hat{t})$.

For an anonymous mechanism to be incentive compatible it must satisfy the incentive constraint and the voluntary participation constraint. By imposing voluntary participation, we assume that each agent has a veto power and hence the decision whether to operate the plant has to be unanimous.

$$
\begin{array}{rll}
E\left[x_{i}\left(t_{-i}, t_{i}\right)-t_{i} \cdot q\left(t_{-i}, t_{i}\right) \mid t_{i}\right] & \\
\quad \geqslant E\left[x_{i}\left(t_{-i}, t_{i}^{\prime}\right)-t_{i} \cdot q\left(t_{-i}, t_{i}^{\prime}\right) \mid t_{i}\right] & \forall t_{i}, t_{i}^{\prime} \in \mathscr{T}_{i} ; \\
E\left[x_{i}\left(t_{-i}, t_{i}\right)-t_{i} \cdot q\left(t_{-i}, t_{i}\right) \mid t_{i}\right] \geqslant 0 & \forall t_{i} \in \mathscr{T}_{i} .
\end{array}
$$

There are two possible feasibility constraints that we can impose on mechanisms. The first, ex ante budget balance, assumes that the mediator has access to risk neutral credit markets. The second, ex post budget balance, assumes that the mediator does not have access to risk neutral credit markets.

$$
\begin{aligned}
E\left[R(n) \cdot q(t)-\sum_{i=1}^{n} x_{i}(t)\right] & \geqslant 0 ; \\
q(t) R(n)-\sum_{i=1}^{n} x_{i}(t) & \geqslant 0 \quad \forall t \in \mathscr{T} .
\end{aligned}
$$

An anonymous mechanism satisfies limited liability if the transfer payments are non-negative:

$$
x_{i}(t) \geqslant 0 \text {. }
$$

An anonymous mechanism is ex post efficient if it provides the project when it is socially optimal:

$$
q(t)= \begin{cases}1, & \text { if } \quad R(n) \geqslant \sum_{i=1}^{n} t_{i} ; \\ 0, & \text { otherwise. }\end{cases}
$$


In Section 3 we characterize mechanisms satisfying ex ante budget balance, and in Section 4 we consider ex post budget balance.

\section{EX ANTE BUDGET BALANCE}

In this section mechanisms are characterized under ex ante budget balance. We first consider mechanisms with the restriction that the type space consists of only two elements, $\mathscr{T}_{i}=\{0,1\}$. This simplification of the type space allows us to derive results in a simple and intuitive way and permits a richer characterization of mechanisms. We first study mechanisms with finitely many agents and we examine their properties, as the number of agents increases. Necessary and sufficient conditions for implementable mechanisms are given in Theorem 1. We then solve for two specific mechanisms: Theorem 2 derives the scheme that maximizes the probability of provision, and Theorem 3 describes the mechanism that maximizes expected profits to the firm. Next, we examine the properties of mechanisms as the number of agents increases. Theorem 4 gives a sufficient condition for mechanisms to satisfy ex post efficiency in large economies. Corollary 1 establishes that the two specific mechanisms studied, the profit-maximizing and the probability-maximizing schemes, satisfy this condition. Thus, in large economies, ex post efficiency can be achieved. We conclude this section with an example, that characterizes the efficiency boundary. In Section 3.2 the main result, that, as the number of agents increases, efficiency is achieved, is established for the general type space.

\subsection{Two Types}

In this section the type space consists of two elements, $\mathscr{T}_{i} \in\{0,1\}$. It is assumed that the conditional density $g\left(t_{i} \mid s\right)$ can be written as $t_{i}=1$ with probability $s$ and $t_{i}=0$ with probability $1-s$. In compact notation this can be written as,

Assumption (iii). $\quad g\left(t_{i} \mid s\right)=s^{t_{i}}(1-s)^{1-t_{i}}$.

Since the type space consists of only two elements, the number of type one announcements, $k=\sum_{j=1}^{n} \hat{t}_{j}$, is a sufficient statistic for the aggregate information available in the economy. The transfer payments to agent $i$ can be written as, $x\left(\sum_{j=1}^{n} \hat{t}_{j}, \hat{t}_{i}\right), x:\{0,1, \ldots, n\} \times\{0,1\} \rightarrow \mathbf{R}(i=1,2, \ldots, n)$, and the probability of providing the project $q\left(\sum_{j=1}^{n} \hat{t}_{j}\right), q:\{0,1, \ldots, n\} \rightarrow[0,1]$. To abbreviate notation, we will sometimes write the transfer payment to type $i$ as $x_{k m}$, where $k=\sum_{j=1}^{n} \hat{t}_{j}$ and $m=\hat{t}_{i}$. Assumptions (i), (ii), and (iii) determine the distribution of $k=\sum_{j=1}^{n} t_{j}$ and $l=\sum_{j \neq i} t_{j}$. Let $p(k)$ be the 
ex ante density of $k=\sum_{j=1}^{n} t_{j}$, and $p\left(l \mid t_{i}\right)$ be the conditional density of $l=\sum_{j \neq i} t_{j}$ given $t_{i}$.

We next state two lemmas which will be useful in the following. In these we calculate $p(k)$, the ex ante density that there are $k=\sum t_{i}$ type ones, and $p\left(l \mid t_{i}\right)$, the conditional density that there are $l=\sum_{j \neq i} t_{j}$ type ones given $t_{i}$ using the distributional assumptions on the random variables $\left(s, t_{1}, t_{2}, \ldots, t_{n}\right)$.

Lemma 1. The density $p(k)$ and the conditional density $p\left(l \mid t_{i}=\hat{t}_{i}\right)$ are given by

$$
\begin{gathered}
p(k)=\left(\begin{array}{l}
n \\
k
\end{array}\right) \cdot \int_{\mathscr{S}} s^{k} \cdot(1-s)^{n-k} \cdot f(s) d s \\
\text { for } \quad k=0,1, \ldots, n . \\
p\left(l \mid t_{i}=\hat{t}_{i}\right)=\left(\begin{array}{c}
n-1 \\
l
\end{array}\right) \cdot \frac{\int_{\mathscr{S}} s^{l} \cdot(1-s)^{n-l-1} \cdot g\left(\hat{t}_{i} \mid s\right) \cdot f(s) d s}{g\left(\hat{t}_{i}\right)} \\
\text { for } \quad l=0,1, \ldots, n-1 .
\end{gathered}
$$

The proof of Lemma 1 is obvious. The following lemma establishes a relationship between the ex ante probabilities and the conditional probabilities which will be used thereafter. The proof of Lemma 2 is given in the Appendix. In the Lemma $g\left(t_{i}\right)$ denotes $\int_{\mathscr{S}} g\left(t_{i} \mid s\right) \cdot f(s) d s$.

LEMMA 2.

$$
\begin{aligned}
p\left(l-1 \mid \hat{t}_{i}=1\right)=\frac{l}{n \cdot g(1)} \cdot p(l) & \text { for } \quad l=1,2, \ldots, n . \\
p\left(l \mid \hat{t}_{i}=0\right)=\frac{n-l}{n \cdot g(0)} \cdot p(l) & \text { for } \quad l=0,1, \ldots, n-1 .
\end{aligned}
$$

Before proceeding with our analysis, we want to argue that limited liability constitutes a "reasonable" assumption in our model. Myerson, Cremer and McLean, McAfee and Reny have shown in a series of papers on optimal auction design with correlated types that, in the absence of limited liability, incentive constraints are not binding. Their result may be stated in our setting, as follows.

Remark 1. There exists an anonymous incentive compatible mechanism satisfying $(\mathrm{EF})$ and $(\mathrm{AB})$.

We give here a particular efficient mechanism and we show in the Appendix that it satisfies (IC), (VP), and (AB): Let $q(k)$ be given by (EF) 
and let the transfer payments to type zero agents be given by $x_{l 0}=0$ for all $l$. Let the transfer payments to type one agents be given by

$$
\begin{aligned}
x_{n 1} & =\frac{p(0 \mid 0)}{p(0 \mid 0) \cdot p(n-1 \mid 1)-p(0 \mid 1) \cdot p(n-1 \mid 0)} \cdot \sum_{l=1}^{n} p(l-1 \mid 1) \cdot q(l), \\
x_{11} & =\frac{\sum_{l=1}^{n} p(l-1 \mid 1) \cdot q(l)}{p(0 \mid 1)}-\frac{p(n-1 \mid 1)}{p(0 \mid 1)} \cdot x_{n 1},
\end{aligned}
$$

and $x_{l 1}=0$ for all $l=2,3, \ldots, n-1$. This mechanism exploits the differences in the conditional probability of different types and gives type one agents a lottery over the possible outcomes with possibly very large positive (respectively negative) payments. The expected value of this lottery for a type one agent equals his expected disutility, whereas for a type zero agent the expected value is zero. Cremer and McLean have shown that one can always find such transfer schemes as long as there is some correlation between types. The problem with these mechanisms is that, at the point where correlation vanishes, the mechanisms will be discontinuous. In the above mechanism the transfer payments go to infinity; $x_{11}$ goes to minus infinity and $x_{n 1}$ to plus infinity. In our setting, with small economic agents seeking compensation payments, this property of the mechanism is not very appealing. Specifically, we would like the transfer payments to be bounded. One way to proceed is to assume risk aversion. With this assumption the payments cannot be too negative and one might expect "reasonable" results. A second approach is to assume limited liability and to allow only for non-negative transfer payments. This is the approach we are going to pursue.

Theorem 1 gives necessary and sufficient conditions for incentive compatible mechanisms satisfying limited liability and ex ante budget balance. The following lemma, which will be used in Theorem 1, characterizes the minimum expenditures needed for a given probability of provision, $q$, so that (IC0), (VP1), and (LL) are satisfied (we ignore (IC1) and (VP0)). The proof of Lemma 3 is given in the Appendix.

Lemma 3. Suppose $q(k)$ is given. The transfer payments $\left(x_{k 0}, x_{k 1}\right)$ that minimize expenditures subject to (IC0), (VP1), and (LL) are given by $x_{k 1}=0$ for $k \neq n, x_{n 1}=1 /(p(n-1 \mid 1)) \cdot \sum_{l=1}^{n} p(l-1 \mid 1) \cdot q(l), x_{k 0}=0$ for $k \neq 0$, and $x_{00}=(p(n-1 \mid 0)) /(p(0 \mid 0) \cdot p(n-1 \mid 1)) \cdot \sum_{l=1}^{n} p(l-1 \mid 1) \cdot q(l)$. Furthermore the minimal expenditures are given by $\sum_{k=0}^{n} p(k) \cdot q(k)$ $(k / n)[1+g(0) / g(1) \cdot(p(n-1 \mid 0) /(p(n-1 \mid 1)]$.

The optimal transfers payments are of the following qualitative form. Type one agents receive payments only if everybody announces type one. 
Type zero agents receive payments only if everybody announces type zero. The intuition is that conditional on their type, type zero agents find it less likely that a high state occurred than type one agents. Paying ones only in the state where everybody announces one, makes zeros at least inclined to mimic ones. As is mentioned in the proof, the optimal payments to type zero agents can actually be arbitrarily chosen as long as they satisfy (IC0) with equality.

We are now able to characterize anonymous mechanisms satisfying (IC), (VP), (LL), and (AB).

THEOREM 1. If an anonymous mechanism $(x, q)$ satisfies (IC), (VP), $(\mathrm{LL}$,$) and (\mathrm{AB})$, then $q(k)$ satisfies

$$
\sum_{k=0}^{n} p(k) \cdot q(k)\left\{r-\frac{k}{n} \cdot\left[1+\frac{g(0)}{g(1)} \cdot \frac{p(n-1 \mid 0)}{p(n-1 \mid 1)}\right]\right\} \geqslant 0 .
$$

If $q(k)$ is monotone decreasing and satisfies (1), then there exist transfers $x$ such that the anonymous mechanism $(x, q)$ satisfies (IC), (VP), (LL), and (AB).

Proof. “ $\Rightarrow$ "Ex ante budget balance implies that, in per capita terms, $0 \leqslant \sum p(k) \cdot\left[q(k) \cdot r-(n-k) / n \cdot x_{k 0}-(k / n) \cdot x_{k 1}\right]$. From Lemma 3 we know that $\sum p(k)\left[((n-k) / n) x_{k 0}+(k / n) x_{k 1}\right] \geqslant \sum p(k) q(k)(k / n)[1+(g(0) / g(1))$ $(p(n-1 \mid 0)) /(p(n-1 \mid 1))]$. Combining these two inequalities yields (1).

" $\Leftarrow$ " Given $q(k)$, we want to show that the optimal transfers from Lemma 3, $x_{l}^{*}$ solve our problem. Since by construction these transfers satisfy (VP1), (IC0), (LL), and $q(k)$ satisfies (1), we only have to verify that these transfers satisfy (IC1) and (VP0). (VP0) is clearly satisfied since $x_{l 0}^{*} \geqslant 0$. Using $x^{*}$ we can rewrite (IC1) as $0 \geqslant \sum_{l=0}^{n-1} p(l \mid 1)$. $[(p(0 \mid 1)) /(p(0 \mid 0)) \cdot(p(n-1 \mid 0)) /(p(n-1 \mid 1)) \cdot q(l+1)-q(l)]$. Observe that $(p(0 \mid 1)) /(p(0 \mid 0)) \cdot(p(n-1 \mid 0)) /(p(n-1 \mid 1))$ is less than or equal to one since $(p(n-1 \mid 0)) /(p(n-1 \mid 1)) \leqslant(p(l \mid 0)) /(p(l \mid 1)) \forall l<n-1$ (from the proof of Lemma 3 ). Now, since $q(k)$ is monotone decreasing in $k$, (IC1) has to be satisfied. (Notice, though, if $q(k)$ is not monotone then (IC1) need not be satisfied.)

Q.E.D.

Condition (1) is necessary for mechanisms to satisfy (IC), (VP), (LL), and $(\mathrm{AB})$ and it is also sufficient if the probability $q(k)$ is monotone decreasing in $k$. The term on the left hand side of (1) is the expected virtual surplus in per capita terms. This is the expected surplus generated by the provision rule $q$ after the agents' costs have been increased by the amount “ $(k / n)(g(0) / g(1)) \cdot(p(n-1 \mid 0) / p(n-1 \mid 1))$ ". This adjustment yields a smaller expected surplus and is due to the private nature of agents' valuations. In accordance with the literature, we call this the "virtual cost" of 
type zero agents. It is the payment to type zero agents necessary to prevent them from imitating type one agents. Observe that the term $(p(n-1 \mid 0) /$ $p(n-1 \mid 1))$ reflects the amount of correlation among agents' types. If types are independent, that is in the limit as correlation vanishes, this ratio equals one. If types are correlated this ratio will be positive (in the interior of the unit interval) and if types are perfectly correlated this ratio equals zero. Thus, the virtual cost of type zero agents is decreasing in the amount of correlation among agents' types.

The question that naturally arises is whether, as correlation gets small, the virtual cost of type zero agents converges to its respective term in a model with independent types. Remark 2 gives an affirmative answer. This is not surprising since Robert [9] has shown, in the context of auctions, that under ( $\mathrm{LL}$ ) and/or risk aversion the optimal expected gain to the seller is continuous in the space of information distributions. Remark 2 establishes that condition ( 1 ) is equivalent to the respective condition in a model with independent types as we approach the independent types case. In that sense, imposing limited liability creates a consistent extension of the independent private information environment to correlated private information.

Remark 2. Consider a sequence of densities $f_{j}(s)$ that converges to the density of a one point distribution. Specifically, suppose there exists a number $a \in(0,1)$ such that

$$
\lim _{j \rightarrow \infty} \int_{0}^{x} f_{j}(s) d s= \begin{cases}1 & \text { if } x \geqslant a \\ 0 & \text { otherwise }\end{cases}
$$

Then condition (1) converges to the implementability constraint of the independent types environment, as $j \rightarrow \infty$.

Proof. Consider the independent type case: Type one agents receive at least their cost, i.e., $x_{l 1}=1 \cdot q(l)$ for all $l$, while type zero agents receive at least their virtual cost, i.e., $x_{l 0}=1 \cdot q(l+1)$ for all $l$, to prevent them from imitating type one agents. Therefore, expected revenues minus virtual costs are given by $\sum_{0}^{n} p(k) q(k) r-\sum_{0}^{n-1}(n-k) / n p(k) q(k+1)-\sum_{1}^{n}(k / n)$ $p(k) q(k)$. We have to show that condition (1) converges to this expression as the correlation vanishes. In condition (1), the transfers to type one agents equal $(k / n) p(k) q(k)$ and transfers to type zeros agents equal $(g(0) / g(1))(p(n-1 \mid 0) / p(n-1 \mid 1)) \cdot(k+1) / n p(k+1) q(k+1)$. The assumption on the sequence $f_{j}$ implies that $\lim _{j \rightarrow \infty} p(k)=\lim _{j \rightarrow \infty}\left(\begin{array}{c}n \\ k\end{array}\right) \int_{S} s^{k}(1-s)^{n-k}$ $f_{j}(s) d s=\left(\begin{array}{c}n \\ k\end{array}\right) a^{k}(1-a)^{n-k}$. Similarly, in the limit, as $j \rightarrow \infty, p(k+1)=\left(\begin{array}{c}n \\ k+1\end{array}\right)$ $a^{k+1}(1-a)^{n-k-1}$ which yields $p(k+1) / p(k)=(n-k) /(k+1) a /(1-a)$. In addition it follows immediately that $p(n-1 \mid 0) / p(n-1 \mid 1) \rightarrow 1$, as $j \rightarrow \infty$. So in the limit, as $j \rightarrow \infty$, the transfer payments to type zero agents are 
equal to $(1-a) / a \cdot 1 \cdot(k+1) / n \cdot a /(1-a) \cdot(n-k) /(k+1) \cdot q(k+1)$. Canceling yields $(n-k) / n p(k) q(k+1)$, as above.

Q.E.D.

Theorem 2 characterizes the maximum probability of provision. The project is provided if the number of type one announcements is less than or equal to $K^{*}$, where $K^{*}$ is the largest number such that expected revenues are greater than or equal to virtual costs. The optimal transfer payments are those characterized in Lemma 3.

THEOREM 2. Let $M$ be the class of anonymous mechanisms satisfying (IC), (VP), ( $\mathrm{AB})$, and (LL) and let

$$
\left(x^{*}, q^{*}\right) \in \underset{(x, q) \in M}{\operatorname{argmax}} \sum_{k=0}^{n} p(k) q(k)
$$

Then $\sum_{k=0}^{K^{*}-1} p(k) \leqslant \sum_{k=0}^{n} p(k) q^{*}(k) \leqslant \sum_{k=0}^{K^{*}} p(k)$, where $K^{*}$ is the largest integer such that

$$
\sum_{k=0}^{K^{*-1}} p(k)\left[r-\frac{g(1)+g(0) \cdot \frac{p(n-1 \mid 0)}{p(n-1 \mid 1)}}{g(1)} \cdot \frac{k}{n}\right] \geqslant 0 .
$$

Proof. Consider the following problem: $\max _{(q(i))_{0}^{n}} \sum_{k=0}^{n} p(k) \cdot q(k)$ subject to (1) and $q(k) \in[0,1]$. Using a change of variable, i.e., $\tau(k):=p(k) q(k)$, we can rewrite this problem as $\max _{\tau} \sum_{0}^{n} \tau(k)$ subject to

$$
\sum_{k=0}^{n} \tau(k)\left[r-\frac{g(1)+g(0) \cdot \frac{p(n-1 \mid 0)}{p(n-1 \mid 1)}}{g(1)} \cdot \frac{k}{n}\right] \geqslant 0 \quad \text { and } \quad \tau(k) \in[0, p(k)] \text {. }
$$

Observe that the term in square brackets is strictly decreasing in $k$. Hence there exists a $K^{*}$ such that it is optimal to set $\tau(k)=p(k)$ for $k<K^{*}$ and $\tau(k)=0$ for $k>K^{*}$. We express our findings in terms of $q(k)$. Formally, the following $q(\cdot)$ solves our problem:

$$
q(k)=\left\{\begin{array}{lll}
1, & \text { if } & k<K^{*} \\
\in[0,1], & \text { if } & k=K^{*} \\
0, & \text { if } & k>K^{*}
\end{array}\right.
$$

Observe that $q(k)$ is monotone decreasing in $k$ and satisfies (1). From Theorem 1 we know that the mechanism satisfies (IC), (VP), (AB), and (LL).

Q.E.D. 
The mechanism in Theorem 2 is a benchmark for what mechanisms can achieve. Other mechanisms will not perform that well, but are still of interest. In particular, consider the mechanism designed by the firm that operates the polluting plant. Assume that the firm is risk neutral and its objective is to maximize expected profits. The following theorem characterizes its optimal mechanism.

THEOREM 3. Let $M$ be the class of anonymous mechanisms satisfying (IC), (VP), ( $\mathrm{AB})$, and ( $\mathrm{LL})$. If

$$
q(k)= \begin{cases}1, & \text { if } \quad r-\frac{k}{n}\left[1+\frac{g(0)}{g(1)} \frac{p(n-1 \mid 0)}{p(n-1 \mid 1)}\right] \geqslant 0 ; \\ 0, & \text { otherwise, }\end{cases}
$$

and the transfer payments are the expenditure minimizing transfers given in Lemma 3 then $(x, q)$ maximizes profit over all mechanisms $\left(x^{\prime}, q^{\prime}\right) \in M$.

Proof. Per capita profits are given by $\sum_{0}^{n} p(k) q(k)\left[r-(k / n) x_{k 1}-\right.$ $\left.((n-k) / n) x_{k 0}\right]$. Lemma 3 determines the expenditure minimizing transfers for any given $q(k)$. Using these our problem is to $\max _{q} \sum p(k) q(k)$ $[r-(k / n)(1+(g(0) / g(1))(p(n-1 \mid 0) / p(n-1 \mid 1)))]$. Since the term in square brackets is strictly decreasing in $k$, it is optimal to set $q(k)=1$ if $\quad r-(k / n)[1+(g(0) / g(1))(p(n-1 \mid 0) / p(n-1 \mid 1))] \geqslant 0, \quad$ and $q(k)=0$ otherwise. Observe that $q(k)$ is monotone decreasing in $k$ and that per capita profits are non-negative. Theorem 1 implies that the mechanism satisfies (IC), (VP), (AB), and (LL).

Q.E.D.

The interpretation of the profit maximizing scheme is straightforward: Operate the polluting plant if and only if the revenues are greater than or equal to virtual costs. The optimal transfers are the expenditure minimizing payments given in Lemma 3.

Next it is investigated what happens to the probability of providing the project as the number of agents increases. Mailath and Postlewaite [5] and Rob [8] have shown that, in large economies with independent types (in general type spaces), the probability that the good be provided when it should goes to zero. We show that this result is not robust in the following sense: For any amount of correlation between types, the probability that the good be provided when it should goes to one as the number of agents goes to infinity. In this sense, the result of Rob and Mailath and Postlewaite is not a limiting case of a correlated valuations model with limited liability.

Lemma 4 determines what happens to the term in the implementability constraint that reflects the virtual cost of type zero agents. The ratio $p(n-1 \mid 0) / p(n-1 \mid 1)$ declines with $n$ and thus, as the number of agents 
increases, the implementability constraint is relaxed and the project will be provided more often. In the limit, as $n \rightarrow \infty$, the virtual cost of type zero agents vanishes. The reason is that type zero agents find it more unlikely than type one agents that everybody else announces to be a high type agent. In particular, as $n$ increases, the conditional probability that everybody else is a type one agent goes faster to zero for type zero agents than it does for type one agents.

\section{Lemma 4. As $n \rightarrow \infty, p(n-1 \mid 0) / p(n-1 \mid 1) \rightarrow 0$.}

Proof. Pick $\varepsilon$ with $0<\varepsilon<1$. Define the real valued function $k_{n}(s)=$ $[s /(1-(\varepsilon / 2))]^{n}$. Lemma 1 implies that

$$
\begin{aligned}
\frac{p(n-1 \mid 0)}{p(n-1 \mid 1)} & =\frac{\left(\begin{array}{c}
n-1 \\
n-1
\end{array}\right) \frac{\int_{0}^{1}(1-s) s^{n-1} f(s) d s}{g(0)}}{\left(\begin{array}{l}
n-1 \\
n-1
\end{array}\right) \frac{\int_{0}^{1} s s^{n-1} f(s) d s}{g(1)}} \\
& =\frac{\int_{0}^{1}(1-s) k_{n-1}(s) f(s) d s}{\int_{0}^{1} s k_{n-1}(s) f(s) d s} \cdot \frac{g(1)}{g(0)} .
\end{aligned}
$$

The second equality is obtained by canceling and by dividing denominator and numerator by $[1-(\varepsilon / 2)]^{n-1}$. Consider the following inequalities which we will show to be true for all large $n$ :

$$
\begin{aligned}
& \frac{\int_{0}^{1}(1-s) k_{n-1}(s) f(s) d s}{\int_{0}^{1} s k_{n-1}(s) f(s) d s} \cdot \frac{g(1)}{g(0)} \\
& \quad=\frac{\int_{0}^{1-\varepsilon}(1-s) k_{n-1}(s) f(s) d s+\int_{1-\varepsilon}^{1}(1-s) k_{n-1}(s) f(s) d s}{\int_{0}^{1-\varepsilon} s k_{n-1}(s) f(s) d s+\int_{1-\varepsilon}^{1} s k_{n-1}(s) f(s)} \cdot \frac{g(1)}{g(0)} \\
& \quad \leqslant \frac{\varepsilon \int_{1-\varepsilon}^{1} k_{n-1}(s) f(s) d s+\int_{1-\varepsilon}^{1}(1-s) k_{n-1}(s) f(s) d s}{\int_{0}^{1-\varepsilon} s k_{n-1}(s) f(s) d s+\int_{1-\varepsilon}^{1} s k_{n-1}(s) f(s)} \cdot \frac{g(1)}{g(0)} \\
& \quad \leqslant \frac{\varepsilon \int_{1-\varepsilon}^{1} k_{n-1}(s) f(s) d s+\varepsilon \int_{1-\varepsilon}^{1} k_{n-1}(s) f(s) d s}{0+(1-\varepsilon) \int_{1-\varepsilon}^{1} k_{n-1}(s) f(s) d s} \cdot \frac{g(1)}{g(0)} \\
& \quad=\frac{2 \varepsilon \int_{1-\varepsilon}^{1} k_{n-1}(s) f(s) d s}{(1-\varepsilon) \int_{1-\varepsilon}^{1} k_{n-1}(s) f(s) d s} \cdot \frac{g(1)}{g(0)} \\
& =\frac{2 \varepsilon}{1-\varepsilon} \cdot \frac{g(1)}{g(0)}
\end{aligned}
$$


The first equality is obtained by breaking up the range of integration into two disjoint subsets. The second line uses the following argument. The definition of $k_{n}(s)$ implies that for all $s \in[0,1-\varepsilon], k_{n}(s)$ converges to zero, as $n \rightarrow \infty$. Since $k_{n}$ is bounded by 1 on $[0,1-\varepsilon]$ for all $n$, the dominated convergence theorem implies that $\int_{0}^{1-\varepsilon} k_{n-1}(s) f(s) d s \rightarrow 0$. Since $1-s$ is less than 1 , this implies that for any $\gamma>0$ there exist a $N$ such that for $n>N$, $\int_{0}^{1-\varepsilon}(1-s) k_{n-1}(s) f(s) d s<\gamma$. Now, observe that for $s \in[1-(\varepsilon / 2), 1]$, $k_{n}(s) \geqslant 1$ for all $n$. This implies that $\int_{1-\varepsilon}^{1} k_{n-1}(s) f(s) d s \geqslant \int_{1-(\varepsilon / 2)}^{1} 1 \cdot f(s) d s$. By assumption (i), full support of the random variable $s$, the right hand side in this inequality is positive. Combining these two statements implies that for $\gamma=\varepsilon \int_{1-(\varepsilon / 2)}^{1} f(s) d s$, there exists an $N$ such that for all $n \geqslant N$, $\int_{0}^{1-\varepsilon}(1-s) k_{n-1}(s) f(s) d s<\varepsilon \int_{1-(\varepsilon / 2)}^{1} f(s) d s \leqslant \varepsilon \int_{1-\varepsilon}^{1} k_{n-1}(s) f(s) d s$.

The third inequality holds since the numerator is increased and the denominator is decreased. To see this observe that $1-s \leqslant \varepsilon$ for $s \in[1-\varepsilon, 1]$ and thus the numerator is not decreased. The denominator is decreased, since $s \geqslant 1-\varepsilon$ for $s \in[1-\varepsilon, 1]$ and the other integral term is greater than or equal to zero. The fourth equality is obtained by rearranging and the final equality is obtained by canceling.

Since $\varepsilon$ is arbitrary small we have $p(n-1 \mid 0) / p(n-1 \mid 1) \rightarrow 0$, as $n \rightarrow \infty$.

Q.E.D.

Assumption (i), which states that the random variable $s$ has support $[0,1]$, is the important assumption in the Lemma. If the maximum in the support of $s$ is smaller than 1 , the ratio $p(n-1 \mid 0) / p(n-1 \mid 1)$ will still decline as $n$ increases, but it will not vanish in the limit. A similar argument as in the proof yields that if the support of $s$ is $\left[0, s^{*}\right]$ with $s^{*}<1$, then as $n$ goes to infinity, $p(n-1 \mid 0) / p(n-1 \mid 1) \rightarrow\left(1-s^{*}\right) / s^{*} \cdot g(1) / g(0)>0$. So if $s^{*}<1$ then $p(n-1 \mid 0) / p(n-1 \mid 1)$ does not vanish in the limit. (However, $\left(1-s^{*}\right) / s^{*} \cdot g(1) / g(0)$ is less than 1 .)

Theorem 4 gives a sufficient condition for mechanisms in large economies to be ex post efficient, and Corollary 1 points out that this condition applies to the mechanisms characterized in Theorems 2 and 3.

THEOREM 4. Let $(x, q)$ be an incentive compatible anonymous mechanism. If the probability, $q($.$) , satisfies q(k)=1$ if $r \geqslant(k / n)[1+(p(n-1 \mid 0) / p(n-1 \mid 1))$ $(g(0) / g(1))]$ and $q(k)=0$ otherwise, then, as $n \rightarrow \infty$, the probability of provision converges to the probability of provision under the ex post efficient rule.

Proof. Ex post efficiency requires that the project is provided if $(k / n) \leqslant r$ and not provided otherwise. The provision rule in the theorem is given by, $q(k)=1$ if $(k / n) \leqslant r /(1+(p(n-1 \mid 0) / p(n-1 \mid 1))(g(0) / g(1)))$ and $q(k)=0$ otherwise. From Lemma $4 p(n-1 \mid 0) / p(n-1 \mid 1) \rightarrow 0$, as $n \rightarrow \infty$. This 
implies that the provision rule in the theorem converges to the ex post efficient rule, as $n \rightarrow \infty$.

Q.E.D.

COROLlary 1. As $n \rightarrow \infty$, the profit maximizing mechanism satisfies (EF). Furthermore, the probability of provision under the probability maximizing scheme is larger than $(\mathrm{EF})$.

Lemma 4 offers the key insight to this result. The virtual costs of type zero agents are declining with the number of agents in the economy and vanish in the limit as $n \rightarrow \infty$. Economic agents are eventually informationally small. In large economies, the implementability constraint is relaxed and approaches the full information setting. With independent types this reasoning is not valid. The ratio, $p(n-1 \mid 0) / p(n-1 \mid 1)$, remains constant and equal to one for all $n$. The virtual costs do not vanish as $n$ increases, and, as Rob and Mailath and Postlewaite have shown, the implementability constraint is actually worsened and the probability of provision, when it should be provided under full information, goes to zero.

EXAMPLE 1. This example illustrates how many agents are required so that there exists an ex post efficient mechanism. Suppose $s$ is drawn from the beta distribution with parameters $\alpha$ and $\beta$. The beta density is given by $f(s)=s^{\alpha-1}(1-s)^{\beta-1} \cdot \Gamma(\alpha+\beta) /(\Gamma(\alpha) \cdot \Gamma(\beta))$, where $\Gamma(\cdot)$ is the gamma function. Note for $\alpha=\beta=1, f(s)$ is the uniform density on $[0,1]$. The mean of $s$ equals $\alpha /(\alpha+\beta)$ and the variance equals $\alpha \beta /\left((\alpha+\beta)^{2}(\alpha+\beta+1)\right)$.

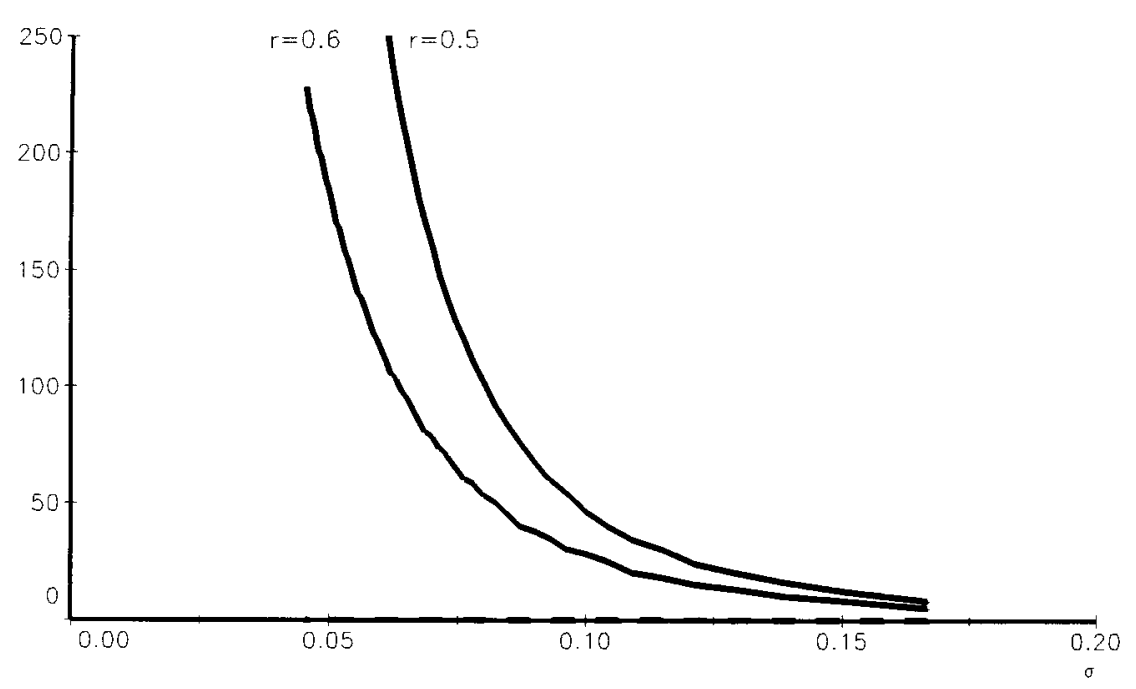

FIG. 1. $n^{*}$ varying with $\sigma, \alpha=\beta$. 
Using that $\Gamma(\alpha+1)=\alpha \cdot \Gamma(\alpha)$ we find that the ratio $p(n-1 \mid 0) / p(n-1 \mid 1)$ equals $\alpha /(\alpha+n-1)$, and hence condition (1) is given by $\sum_{0}^{K^{*}} p(k)[r-(k / n)$ $(1+\alpha /(\alpha+n-1))] \geqslant 0$. We want to calculate the ex post efficiency boundary for a given $r$. Formally, given $\alpha$ and $\beta$, we want to find the smallest $n^{*}$, such that for all $K$ with $K \geqslant r \cdot n^{*}$, it is true that $\sum_{0}^{K} p(k)[r-(k / n)$ $(1+\alpha /(\alpha+n-1))] \geqslant 0$. To simplify the exercise let us fix the mean of the prior of the random variable $s$ at $\frac{1}{2}$ so that $\alpha=\beta$. Figure 1 illustrates the efficiency boundaries for $r=0.5$ and $r=0.6$ respectively. On the horizontal axis is the standard deviation of $s$ which we denote by $\sigma$, and on the vertical axis is the minimal number of agents, $n^{*}$. In the area above the curve there exist mechanisms that satisfy efficiency.

From Fig. 1 we see that as $r$ increases the efficiency boundary shifts to the left. Therefore, for a given $\alpha$, the number of agents $n(r)$ required to achieve efficiency is decreasing in $r$.

\subsection{General Type Space}

In this section, the main result of the Section 3.1 is generalized to finitely many types. We replace Assumption (iii) in Section 3.1 with the following assumption:

Assumption (iii'). For all $\sigma \in \mathscr{T}_{i}$ there exists a state, $s \in \mathscr{S}$, such that $g(\sigma \mid s)=1$.

This assumption states, that for every cost type there exists a state where everybody has that cost. In other words the event that everybody has cost $\sigma$ is possible.

Lemma 5 gives the key result used in Theorem 5. It establishes that, as $n$ increases, the probability of the event that everybody else is of type $\sigma$ goes faster to zero for a type $\tau \neq \sigma$ than for a type $\sigma$. The proof of Lemma 5 is similar to the proof of Lemma 4.

Lemma 5. For all $\sigma, \tau \in \mathscr{T}_{i}$ with $\sigma \neq \tau$,

$$
\frac{\int g(\tau \mid s) \cdot g(\sigma \mid s)^{n-1} \cdot f(s) d s}{\int g(\sigma \mid s)^{n} \cdot f(s) d s} \rightarrow 0, \quad \text { as } \quad n \rightarrow \infty .
$$

Proof. Pick $\varepsilon$ arbitrarily small with $0<\varepsilon<1$ and consider any $\sigma, \tau \in \mathscr{T}_{i}$ with $\sigma \neq \tau$. Let $A=\{s \in \mathscr{S} \mid g(\sigma \mid s) \geqslant 1-\varepsilon\}$ and define the real valued function $k_{n}(s)=[g(\sigma \mid s) /(1-(\varepsilon / 2))]^{n}$. Assumption (iii') implies that the set $A$ is nonempty. Assumption (i), full support of $s$, and Assumption (ii), continuity of $g(\sigma \mid s)$ in $s$, imply that the measure of the set $A$ is positive. We claim that $k_{n}$ converges uniformly to zero on the complement of the set $A$, which we denote by $A^{c}$. Indeed for all $s \in A^{c}$, we have the uniform bound $\left|k_{n}(s)\right| \leqslant((1-\varepsilon) /(1-(\varepsilon / 2)))^{n}$ and $(1-\varepsilon) /(1-(\varepsilon / 2))<1$ which proves our claim. 
We can write

$$
\begin{aligned}
& \frac{\int g(\tau \mid s) g(\sigma \mid s)^{n-1} f(s) d s}{\int g(\sigma \mid s)^{n} f(s) d s} \\
& \quad=\frac{\int_{A^{c}} g(\tau \mid s) k_{n-1}(s) f(s) d s+\int_{A} g(\tau \mid s) k_{n-1}(s) f(s) d s}{\int_{A^{c}} g(\sigma \mid s) k_{n-1}(s) f(s) d s+\int_{A} g(\sigma \mid s) k_{n-1}(s) f(s) d s}
\end{aligned}
$$

by dividing both numerator and denominator by $(1-(\varepsilon / 2))^{n-1}$ and using $A \cup A^{c}=\mathscr{S}$.

By the dominated convergence theorem $\int_{A^{c}} g(\tau \mid s) k_{n-1}(s) f(s) d s \rightarrow 0$, as $n \rightarrow \infty$, since $k_{n-1}$ converges to zero on $A^{c}$ and $g(\tau \mid s)$ is bounded by 1 . On the other hand $\int_{A} k_{n-1}(s) f(s) d s$ does not converge to zero since the set $\{s \mid g(\sigma \mid s) \geqslant 1-(\varepsilon / 2)\}$ contained in $A$ has positive measure and $k_{n}(s) \geqslant 1$ on this set. Thus there exists an $N$ such that for all $n \geqslant N$, $\int_{A^{c}} g(\tau \mid s) k_{n-1}(s) f(s) d s<\varepsilon \int_{A} k_{n-1}(s) f(s) d s$.

Observe that by definition of the set $A, g(\tau \mid s) \leqslant \varepsilon$ and $g(\sigma \mid s) \geqslant 1-\varepsilon$ for all $s \in A$. Using these two statements implies that for all $n \geqslant N$ the following inequality is satisfied:

$$
\begin{aligned}
& \frac{\int_{A^{c}} g(\tau \mid s) k_{n-1}(s) f(s) d s+\int_{A} g(\tau \mid s) k_{n-1}(s) f(s) d s}{\int_{A^{c}} g(\sigma \mid s) k_{n-1}(s) f(s) d s+\int_{A} g(\sigma \mid s) k_{n-1}(s) f(s) d s} \\
& \quad \leqslant \frac{\varepsilon \int_{A} k_{n-1}(s) f(s) d s+\int_{A} \varepsilon k_{n-1}(s) f(s) d s}{0+\int_{A}(1-\varepsilon) k_{n-1}(s) f(s) d s} \\
& \quad=\frac{2 \varepsilon \int_{A} k_{n-1}(s) f(s) d s}{(1-\varepsilon) \int_{A} k_{n-1}(s) f(s) d s} \\
& \quad=\frac{2 \varepsilon}{1-\varepsilon}
\end{aligned}
$$

The first inequality is satisfied if the numerator increases and the denominator decreases. The numerator increases, since $\int_{A^{c}} g(\tau \mid s) k_{n-1}(s) f(s) d s<$ $\varepsilon \int_{A} k_{n-1}(s) f(s) d s$ and $g(\tau \mid s) \leqslant \varepsilon$ for all $s \in A$. The denominator decreases since $\int_{A^{c}} g(\sigma \mid s) k_{n-1}(s) f(s) d s \geqslant 0$ and $g(\sigma \mid s) \geqslant 1-\varepsilon$ for all $s \in A$. The second and third equality are obtained by rearranging and canceling. Since $\varepsilon$ is arbitrarily small we have $\int g(\tau \mid s) g(\sigma \mid s)^{n-1} f(s) d s / \int g(\sigma \mid s)^{n} f(s) d s \rightarrow 0$, as $n \rightarrow \infty$.

Q.E.D.

The following theorem is the main result of this section.

THEOREM 5. There exists an $N$ such that for all $n>N$ there exist anonymous mechanisms satisfying ( $\mathrm{IC}),(\mathrm{VP}),(\mathrm{LL})$, and $(\mathrm{AB})$ that provide the project when it is ex post efficient. 
The theorem states that ex post efficiency is achievable for large $n$. To prove the theorem we construct an ex post efficient mechanism and show that it satisfies (IC), (VP), (LL), and (AB) for all large $n$.

Proof. We construct a mechanism and show that this mechanism has the desired properties. Let

$$
q(t)= \begin{cases}1 & \text { if } \frac{1}{n} \sum t_{i} \leqslant r \\ 0 & \text { otherwise. }\end{cases}
$$

be the ex post efficient provision rule. Consider transfers to agent $i$ of type $\sigma \in \mathscr{T}_{i}$ such that the agent receives his expected cost of pollution in the event where everybody announces to be of type $\sigma$. The transfer payments can be written as

$$
\bar{x}_{\sigma}(\hat{t})= \begin{cases}\sigma \frac{E[q(t) \mid \sigma]}{E\left[1_{\left\{t^{\prime} \in \mathscr{T} \mid t_{j}^{\prime}=\sigma, \forall j \neq i\right\}}\left(t_{-i}, \sigma\right) \mid \sigma\right]} & \text { if } \hat{t}=(\sigma, \sigma, \ldots, \sigma) \\ 0 & \text { otherwise; }\end{cases}
$$

where $1_{A}(t)$ denotes the characteristic function of $A$; that is, $1_{A}(t)=1$ if $t \in A$, and $1_{A}(t)=0$ otherwise. For some types, for instance type $\sigma=0$, the transfers $\bar{x}_{\sigma}$ equal 0 . In addition to types, with $\lim _{n \rightarrow \infty} E\left[\bar{x}_{\sigma}(t) \mid \sigma\right]=0$, the expected transfers eventually vanish. Since these agents may gain from announcing a different type, we pay them the maximum between their own $E\left[\bar{x}_{\sigma}(t) \mid \sigma\right]$ and the expected payment obtained by mimicking any other type. We denote by $E\left[\bar{x}_{\tau}\left(t_{-i}, \tau\right) \mid \sigma\right]$ the expected transfer payment received by an agent of type $\sigma$ who announces $\tau$ and we sometimes abbreviate this transfer payment by $E\left[\bar{x}_{\tau} \mid \sigma\right]$. Formally, we split the set of types $\mathscr{T}_{i}$ into two disjoint subsets $\mathscr{T}^{1}$ and $\mathscr{T}^{2}$, where $\mathscr{T}^{2}=\left\{\sigma \in \mathscr{T}_{i} \mid\right.$ $\left.\lim _{n \rightarrow \infty} E\left[\bar{x}_{\sigma} \mid \sigma\right]=0\right\}$ and $\mathscr{T}^{1}=\mathscr{T}_{i}-\mathscr{T}^{2}$. We define transfers to agents of type $\sigma \in \mathscr{T}^{1}$ as $x_{\sigma}(t)=\bar{x}_{\sigma}(t)$. For agents with type $\sigma \in \mathscr{T}^{2}$ the transfers are defined as the maximum between their own $\bar{x}_{\sigma}(t)$, the expected payment obtained by mimicking types in $\mathscr{T}_{i}$ and the expected payment received by any type $\tau^{\prime} \in \mathscr{T}^{2}$. Specifically we define the transfers for agents with type $\sigma \in \mathscr{T}^{2}$ as

$$
x_{\sigma}(\hat{t})= \begin{cases}\frac{\max _{\tau \in \mathscr{T}_{i}, \tau^{\prime} \in \mathscr{T}^{2}} E\left[\bar{x}_{\tau}\left(t_{-i}, \tau\right) \mid \tau^{\prime}\right]}{E\left[1_{\left\{t^{\prime} \in \mathscr{T} \mid t_{j}^{\prime}=\sigma, \forall j \neq i\right\}}\left(t_{-i}, \sigma\right) \mid \sigma\right]} & \text { if } \hat{t}=(\sigma, \sigma, \ldots, \sigma) ; \\ 0 & \text { otherwise. }\end{cases}
$$

In this definition the maximum is also taken over $\tau^{\prime} \in \mathscr{T}^{2}$ to ensure that an agent of type $\sigma \in \mathscr{T}^{2}$ receives at least as much as any type $\tau^{\prime} \in \mathscr{T}^{2}$. As will 
be shown below, this is used to rule out that types in the set $\mathscr{T}^{2}$ can profitably mimick other types in the set $\mathscr{T}^{2}$.

We have to show that there exists an $N$ such that for all $n>N$ this mechanism satisfies (IC), (VP), (LL), and (AB). First, observe that (LL) is satisfied, since $x_{\sigma}(t) \geqslant 0$. Second, observe that (VP) is satisfied. This follows from the definition of the transfer payments $x_{\sigma}$, since for agents with type $\sigma \in \mathscr{T}^{1}$ the expected payment $x_{\sigma}$ exactly equals their expected loss; that is, $E\left[x_{\sigma}(t) \mid \sigma\right]=\sigma E[q(t) \mid \sigma]$. For agents with type $\sigma \in \mathscr{T}^{2}$ the expected payments are at least as large as the expected loss since $E\left[x_{\sigma} \mid \sigma\right] \geqslant E\left[\bar{x}_{\sigma} \mid \sigma\right]=\sigma E[q \mid \sigma]$. This implies (VP). Next, it is shown that (IC) is satisfied. Consider first agents of type $\sigma \in \mathscr{T}^{2}$. By definition their expected payment is at least as large as the maximum payment they could receive by announcing any other type $\tau \in \mathscr{T}^{1}$. This ensures that (IC) is satisfied with respect to announcements in the set $\mathscr{T}^{1}$. In addition they receive at least as much as any other type in the set $\mathscr{T}^{2}$. Now mimicking a type $\tau^{\prime}$ in the set $\mathscr{T}^{2}$ eventually yields a smaller expected payoff than reporting truthfully. To see this observe that the expected payment received by mimicking a type $\tau^{\prime}$ agent is given by $\int g\left(\tau^{\prime} \mid s\right)^{n-1} g(\sigma \mid s) f(s) d s / \int g\left(\tau^{\prime} \mid s\right)^{n} f(s) d s \cdot g\left(\tau^{\prime}\right) / g(\sigma) \max _{\tau \in \mathscr{T}_{i}, \sigma^{\prime} \in \mathscr{T}^{2}} E\left[\bar{x}_{\tau} \mid \sigma^{\prime}\right]$ $\leqslant \max _{\tau \in \mathscr{T}_{i}, \sigma^{\prime} \in \mathscr{T}^{2}} E\left[\bar{x}_{\tau} \mid \sigma^{\prime}\right]$ for large $n$, since from Lemma 5 the first term on the left hand side of the inequality vanishes as $n$ increases. This implies that there exists an $N$ such that for all $n>N$ (IC) is satisfied for types in the set $\mathscr{T}^{2}$. For agents of type $\sigma \in \mathscr{T}^{1}$ the (IC) constraint can be written as:

$$
E\left[x_{\sigma} \mid \sigma\right]-\sigma E[q \mid \sigma] \geqslant E\left[x_{\tau}\left(t_{-i}, \tau\right) \mid \sigma\right]-\sigma \cdot E\left[q\left(t_{-i}, \tau\right) \mid \sigma\right] .
$$

The left hand side of this inequality equals zero, since $E\left[x_{\sigma} \mid \sigma\right]=\sigma E[q \mid \sigma]$. The right hand side is negative for sufficiently large $n$. To see this, we show that the first term in the right hand side vanishes and the second term in the right hand side converges to a negative number as $n$ increases.

Consider first the second term on the right hand side. By Assumption (i), $\tau \leqslant T$ is finite, and the announcement of an individual agent has eventually no effect on the probability of provision, as $T / n \rightarrow 0$, as $n \rightarrow \infty$. Therefore, as $n$ increases, the term $\sigma \cdot E\left[q\left(t_{-i}, \tau\right) \mid \sigma\right]$ approaches $\sigma \cdot E\left[q\left(t_{-i}, \sigma\right) \mid \sigma\right]$, which converges to a positive number, as $n \rightarrow \infty$, by definition of the set $\mathscr{T}^{1}$. (The $\lim _{n \rightarrow \infty} E[q \mid \sigma]$ exists and can be calculated using the law of large numbers. Let $A(\sigma)=\left\{s \in S \mid \sum_{\sigma \in \mathscr{T}_{i}} \sigma g(\sigma \mid s) \leqslant r\right\}$ be the set of states in which the expected cost of pollution is smaller than the revenues $r$. By the law of large numbers the conditional probability of provision $E[q \mid \sigma]$ converges to $\int_{A(\sigma)} h(s \mid \sigma) d s$, as $n \rightarrow \infty$.)

Consider next the first term on the right hand side. We distinguish two cases: an announcement $\tau \in \mathscr{T}^{1}$ and an announcement $\tau \in \mathscr{T}^{2}$. We begin 
with an announcement $\tau \in \mathscr{T}^{2}$. In this case the first term on the right hand side is given by

$$
\frac{\int g(\tau \mid s)^{n-1} g(\sigma \mid s) f(s) d s}{\int g(\tau \mid s)^{n} f(s) d s} \cdot \frac{g(\tau)}{g(\sigma)} \cdot \max _{\sigma^{\prime} \in \mathscr{T}_{i}, \tau^{\prime} \in \mathscr{T}^{2}} E\left[\bar{x}_{\sigma^{\prime}}\left(t_{-i}, \sigma^{\prime}\right) \mid \tau^{\prime}\right] .
$$

From Lemma 5, the first term in this expression approaches zero as $n$ increases. The second term also approaches 0 as $n$ increases. To see this, observe that for $\tau^{\prime}=\sigma^{\prime}$ the term equals $E\left[\bar{x}_{\tau^{\prime}} \mid \tau^{\prime}\right]$ which approaches 0 by definition of the set $\mathscr{T}^{2}$. For $\tau^{\prime} \neq \sigma^{\prime}$ the term is given by $\int g\left(\sigma^{\prime} \mid s\right)^{n-1}$ $g\left(\tau^{\prime} \mid s\right) f(s) d s / \int g\left(\sigma^{\prime} \mid s\right)^{n} f(s) d s \cdot g\left(\sigma^{\prime}\right) / g\left(\tau^{\prime}\right) \cdot \sigma^{\prime} \cdot E\left[q\left(t_{-i}, \sigma^{\prime}\right) \mid \sigma^{\prime}\right]$ which approaches 0 by Lemma 5. Consider next the second case. For an announcement $\tau \in \mathscr{T}^{1}$, the first term on the right hand side of the (IC) constraint is given by $\int g(\tau \mid s)^{n-1} g(\sigma \mid s) f(s) d s / \int g(\tau \mid s)^{n} f(s) d s \cdot g(\tau) / g(\sigma) \cdot \tau$. $E\left[q\left(t_{-i}, \tau\right) \mid \tau\right]$. Again from Lemma 5 the first part in this expression approaches zero as $n$ increases. So there exists an $N$ such that for $n>N$ the right hand side is negative, and the (IC) constraint will be satisfied.

It is left to show (AB). First, consider the expected sum of the transfer payments to agents announcing a type $\sigma \in \mathscr{T}^{1}$ :

$$
\begin{aligned}
E \sum_{i, \sigma \in \mathscr{T}^{1}} x_{\sigma}(t) & =E \sum_{i, \sigma \in \mathscr{T}^{1}} E\left[x_{\sigma}(t) \mid \sigma\right] \\
& =E \sum_{i, \sigma \in \mathscr{T}^{1}} E\left[\sigma \cdot q\left(t_{-i}, \sigma\right) \mid \sigma\right] \\
& =E\left[q(t) \sum_{i, t_{i} \in \mathscr{T}^{1}} t_{i}\right] .
\end{aligned}
$$

The first equality is obtained by the law of iterated expectations. The second equality follows from the definition of $x_{\sigma}(t)$. The third equality is obtained by the law of iterated expectations and replacing $\sigma$ with $t_{i}$. Next it is shown that the payment to agents who announce a type $\sigma \in \mathscr{T}^{2}$ approaches 0 as $n$ increases. The expected payment, $E x_{\sigma}(t)$, is given by

$$
\begin{aligned}
& E {\left[\max _{\tau \in \mathscr{T}_{i}} \max _{\tau^{\prime} \in \mathscr{T}^{2}} E\left[\bar{x}_{\tau}\left(t_{-i}, \tau\right) \mid \tau^{\prime}\right]\right] } \\
&=E\left[\operatorname { m a x } \left(\max _{\tau^{\prime} \in \mathscr{T}^{2}} E\left[\bar{x}_{\tau^{\prime}}(t) \mid \tau^{\prime}\right],\right.\right. \\
& \\
&\left.\left.\max _{\tau \in \mathscr{T}_{i}, \tau^{\prime} \in \mathscr{T}^{2}, \tau^{\prime} \neq \tau} \frac{\int g\left(\tau^{\prime} \mid s\right) g(\tau \mid s)^{n-1} f(s) d s}{\int g(\tau \mid s)^{n} f(s) d s} \cdot \frac{g(\tau)}{g\left(\tau^{\prime}\right)} \cdot \tau \cdot E[q(t) \mid \tau]\right)\right] .
\end{aligned}
$$

The first expression in the max approaches 0 as $n$ increases, from the definition of the set $\mathscr{T}^{2}$. The second term in the max approaches 0 as $n$ increases, from Lemma 5 . Thus for $\delta>0$ arbitrarily small there exists 
$N$ such for all $n>N, E x_{\sigma}(t)<\delta \cdot E q(t)$. Using $E \sum_{i, \sigma \in \mathscr{T}^{1}} x_{\sigma}(t)=E q(t)$ $\sum_{i, t_{i} \in \mathscr{T}^{1}} t_{i}$ and for agents with $\sigma \in \mathscr{T}^{2}, E x_{\sigma}(t)<\delta \cdot E q(t)$, the (AB) constraint can be written in per capita terms as:

$$
E\left[q(t)\left(r-\frac{1}{n} \sum_{t_{i} \in \mathscr{T}^{1}} t_{i}-\delta\right)\right] \geqslant 0 .
$$

This inequality is satisfied since expected revenues minus expected costs are strictly positive. To see this, observe that by Assumptions (ii) and (iii'), for any $\varepsilon>0$, there exists a neighborhood $U\left(s_{0}\right)$ around the state $s_{0}$, in which type 0 agents occur with probability one, such that for all $s \in U\left(s_{0}\right)$, $g(0 \mid s) \geqslant 1-\varepsilon$. Since for $t_{i}>0, g\left(t_{i} \mid s\right) \leqslant \varepsilon$ and $t_{i} \leqslant T$, the law of large numbers implies that $\lim _{n \rightarrow \infty}(1 / n) \sum t_{i} \leqslant \varepsilon T$ with probability one for all $s \in U\left(s_{0}\right)$. For $\varepsilon$ sufficiently small, that is, for $\varepsilon \leqslant r /(2 T)$, the per capita costs are less than $r / 2$ and therefore the revenues strictly exceed the costs on $U\left(s_{0}\right)$. Due to Assumption (i), full support of $s$, this neighborhood $U\left(s_{0}\right)$ has positive probability. Moreover, since the per capita costs are less than $r / 2$ the efficient probability of provision includes the neighborhood $U\left(s_{0}\right)$ in the limit, as $n \rightarrow \infty$. This implies that there exist $\gamma>0$ and $N$ such that for $n>N, E\left[q(t)\left(r-(1 / n) \sum t_{i}\right)\right] \geqslant \gamma$. Picking $\delta=\gamma$ yields $E[q(t)(r-(1 / n)$ $\left.\left.\sum t_{i}-\delta\right)\right] \geqslant 0$ and $(\mathrm{AB})$ is eventually satisfied.

Q.E.D.

Theorem 5 says that as the number of agents increases the free rider problem is relaxed. For sufficiently many agents the free rider problem vanishes completely. Mechanisms can be implemented that provide the project when it is ex post efficient.

The intuition for this result can be illustrated by two effects. Both effects arise as the number of agents increases. The first effect is that the announcement of an individual agent has a negligible effect on the probability of provision. The second effect is that the expected gain of lying, and announcing a different type, decreases and vanishes in the limit.

To see the second effect, suppose initially that states where everybody is of a particular type occur with positive probability. Define transfers to agents of type $\sigma$ equaling the expected cost of pollution for type $\sigma$ and paid only in the event where everybody announces to be of type $\sigma$. These transfers are bounded since by assumption agents of type $\sigma$ always assign positive probability to this event. Now consider an agent of a different type, say $\tau \neq \sigma$. This agent knows for sure that the state where everybody is of type $\sigma$ did not occur. So, with sufficiently many agents, this agent will assign arbitrary small probability to the event that everybody else is of type $\sigma$. Therefore, the expected gain from lying, and mimicking a type $\sigma$, vanishes.

This illustration relied on the assumption that some states occur with positive probability. Without it, the argument is a little more complicated. 
Essentially, the argument in the proof uses the fact that the conditional probability of the event that all other agents are of type $\sigma$ goes faster to zero for a type $\tau$ than for a type $\sigma$, as shown in Lemma 5 .

Under independent private information the second effect is not present, since the posterior distribution after observing the own type is the same for all types. Mechanisms cannot exploit differences in conditional probabilities. Only the first effect, that eventually the announcement of an individual agent has a negligible effect on the probability of provision, remains. As the number of agents increases, the incentive constraint is actually worsened and it becomes impossible to achieve efficiency as Rob [8] and Mailath and Postlewaite [5] have shown.

The difference between independent and correlated information is also reflected in the bargaining position of an individual agent. Under independence, observing a type does not help predicting another agent's type. Private information is complementary. The bargaining position of individual agents does not get weaker as the number of agents increases. With correlation, observing a type improves the inference about other agents' types. The inference gets more accurate as the number of observed types increases. Private information is substitutable and an increase in the number of agents yields a weakening of the bargaining position of individual agents.

The mechanisms studied in this section rely on transfer payments to agents only if all agents announce to be of the same type. The mediator receives revenues when the probability of provision is positive and incurs costs only in a certain state, independent of whether the project is actually provided or not. In other words, the mediator receives a lottery with positive and negative payments. This does not pose any problems if the project at hand is small in size relative to the overall budget of the government or firm, and when there is access to a risk neutral credit market. But still one might be interested in situations where there is no risk free credit market or might at least want to decrease the variance in the lottery. An extreme way to do so is to impose ex post budget balance. This not only removes all the risk from the mediator, but also puts an upper bound on the feasible transfer payments.

\section{EX POST BUDGET BALANCE}

Next it is established that even under ex post budget balance for a sufficiently large number of agents the probability of provision is positive. We restrict the type space to consist of two elements, $\mathscr{T}_{i}=\{0,1\}$, and adopt the same formulation as in Section 3.1. We assume that the conditional density $g\left(t_{i} \mid s\right)$ can be written as $t_{i}=1$ with probability $s$, and $t_{i}=0$ with 
probability $1-s$ (assumption (iii) in Section 3.1). Since the type space consists of two elements, the number of type one announcements, $k=\sum_{j=1}^{n} \hat{t}_{j}$, is a sufficient statistic for the aggregate information available and the transfer payments, and the probability of provision can be redefined to be a function of $k$ and $t_{i}$. The distribution of $k$ is determined by assumptions (i), (ii), and (iii). We denote by $p(k)$ the ex ante density of $k=\sum_{j=1}^{n} \hat{t}_{j}$ and by $p\left(l \mid t_{i}\right)$ the conditional ex ante density of $l=\sum_{j \neq i} \hat{t}_{j}$ given $t_{i}$. Lemmas 1 and 2 in Section 3.1 characterize $p(k)$ and $p\left(l \mid t_{i}\right)$.

The following theorem states that for sufficiently many agents the probability of provision is positive. The proof of the theorem is given in the appendix.

THEOREM 6. Let $\hat{M}$ be the class of anonymous mechanism satisfying (IC), (VP), (PB) and (LL) and let $\left(x^{*}, q^{*}\right) \in \operatorname{argmax}_{(x, q) \in \hat{M}} \sum_{k=0}^{n} p(k) q(k)$. There exists an $N$ such that for all $n>N$ the probability of provision, $\sum_{k=0}^{n} p(k) q^{*}(k)$, is positive.

In the proof we give a specific mechanism and show that the probability of provision under this scheme is positive. This implies that the optimal mechanism also achieves a positive probability of provision. The mechanism constructed is of the following qualitative form. The project is provided if the fraction of type ones falls into one of two separate intervals: $[0, \varepsilon]$ and $[r / 2, r / 2+\delta]$. In the interval $[0, \varepsilon]$ all the revenues go to type zeros. In the interval $[r / 2, r / 2+\delta]$ all the revenues go to type ones. Observe that this scheme satisfies (LL) and (PB). In the proof of the theorem it is established that (VP) and (IC) are satisfied for $\delta$ and $\varepsilon$ sufficiently small. The intuition is the following. Similar to the previous section, zeros will assign more probability to the event that a small fraction of agents are of type one than ones. For appropriately chosen $\delta, \varepsilon$ expected payments in the interval $[0, \varepsilon]$ are larger for zeros than for ones. Similarly expected payments in the interval $[r / 2, r / 2+\delta]$ are higher for type ones than for zeros, implying (IC). In addition, for $\delta, \varepsilon$ small (VP) is satisfied. Intuitively, around $r / 2$ every type one agent receives transfers of 2 , compensating for the additional loss in region $[0, \varepsilon]$, in which everything is paid to zeros.

This construction relies on the assumption that there is some correlation between types. As correlation decreases, the above regions decrease as well. In the limit they vanish completely, and the probability of provision in the constructed mechanism is zero. ${ }^{4}$

\footnotetext{
${ }^{4}$ In a working paper version of the paper, it is shown that in the optimal mechanism the probability of provision also vanishes as correlation goes to zero. Specifically, under the assumption that $s$ is drawn from a beta distribution a bound for the optimal probability of provision is characterized. Then it is shown that in the limit in which first the number of agents increases and then correlation vanishes, this bound goes to zero. So under ex post budget balance the order of limits does not matter.
} 


\section{CONCLUSION}

We have examined in a simple model the properties of mechanisms under the presence of negative externalities when agents types are correlated. Under the assumption of limited liability and ex ante budget balance, we found that the implementability constraint is similar to the independence case, except for the fact that the virtual utilities are diminished by a factor due to the correlation of agents' types. Furthermore, this factor increases as the number of agents increases and therefore the project will be provided more often.

In large economies, we found that, if there is some correlation between agents types, then the project will be provided when it is socially efficient. In particular, the mechanism that maximizes the expected profits of the firm operating the polluting plant is efficient. Thus the free rider problem is relaxed as the number of agents increases and vanishes for sufficiently many agents.

Even under ex post budget balance in large economies, we found that, if there is some correlation between agents' types, then the probability of provision will be positive.

\section{APPENDIX}

Proof of Lemma 2.

$$
\frac{p(l \mid 0)}{(n-l) \cdot p(l)}=\frac{\left(\begin{array}{c}
n-1 \\
l
\end{array}\right) \cdot \frac{\int_{\mathscr{S}} g(1 \mid s)^{l} \cdot g(0 \mid s)^{n-l} \cdot f(s) d s}{g(0)}}{(n-l) \cdot\left(\begin{array}{l}
n \\
l
\end{array}\right) \cdot \int_{\mathscr{S}} g(1 \mid s)^{l} \cdot g(0 \mid s)^{n-l} \cdot f(s)} d s .
$$

Canceling $\int_{\mathscr{S}} g(1 \mid s)^{l} \cdot g(0 \mid s)^{n-l} \cdot f(s)$ yields

$$
\begin{aligned}
= & \frac{(n-1) !}{(n-1-l) ! l !} \cdot \frac{1}{g(0)} \\
\frac{(n-l) \cdot n !}{(n-l) ! l !} & \frac{1}{n \cdot g(0)} ; \\
\frac{p(l-1 \mid 1)}{l \cdot p(l)}= & \frac{\left(\begin{array}{c}
n-1 \\
l-1
\end{array}\right) \cdot \frac{\int_{\mathscr{S}} g(1 \mid s)^{l} \cdot g(0 \mid s)^{n-l} \cdot f(s) d s}{g(1)}}{l \cdot\left(\begin{array}{c}
n \\
l
\end{array}\right) \cdot \int_{\mathscr{S}} g(1 \mid s)^{l} \cdot g(0 \mid s)^{n-l} \cdot f(s) d s} .
\end{aligned}
$$


Canceling $\int_{\mathscr{S}} g(1 \mid s)^{l} \cdot g(0 \mid s)^{n-l} \cdot f(s) d s$ yields

$$
=\frac{\frac{(n-1) !}{(n-l) !(l-1) !} \cdot \frac{1}{g(1)}}{\frac{l \cdot n !}{(n-l) ! l !}}=\frac{1}{n \cdot g(1)} .
$$

Proof of Remark 1. We give here a particular solution to the above problem, and we show that it satisfies (IC), (VP), (AB) and (EF). Let the transfer payments to type 0 be given by,

$$
x_{l 0}=0, \quad \text { for all } l,
$$

and also let the transfer payments to type 1 agents be given by

$$
\begin{aligned}
& x_{n 1}=\frac{p(0 \mid 0)}{p(0 \mid 0) \cdot p(n-1 \mid 1)-p(0 \mid 1) \cdot p(n-1 \mid 0)} \cdot \sum_{l=1}^{n} p(l-1 \mid 1) \cdot q(l), \\
& x_{11}=\frac{\sum_{l=1}^{n} p(l-1 \mid 1) \cdot q(l)}{p(0 \mid 1)}-\frac{p(n-1 \mid 1)}{p(0 \mid 1)} \cdot x_{n 1},
\end{aligned}
$$

and

$$
x_{l 1}=0 \quad \text { for all } l=2,3, \ldots, n-1 .
$$

(A1) implies that (VP0) and (IC1) are satisfied. (A3) and (A4) imply that (VP1) holds with equality. (A4) implies that (IC0) can be written as:

$$
0 \geqslant p(0 \mid 0) \cdot x_{11}+p(n-1 \mid 0) \cdot x_{n 1} .
$$

Using (A3) yields,

$$
=\frac{p(0 \mid 0)}{p(0 \mid 1)} \cdot\left[\sum_{l=1}^{n} p(l-1 \mid 1) \cdot q(l)-p(n-1 \mid 1) \cdot x_{n 1}\right]+p(n-1 \mid 0) \cdot x_{n 1} .
$$

Using (A2) this yields,

$$
\begin{aligned}
= & \frac{p(0 \mid 0)}{p(0 \mid 1)} \cdot \sum_{l=1}^{n} p(l-1 \mid 1) \cdot q(l)-\left[\frac{p(0 \mid 0)}{p(0 \mid 1)} \cdot p(n-1 \mid 1)-p(n-1 \mid 0)\right] \\
& \cdot \frac{p(0 \mid 0)}{p(0 \mid 0) \cdot p(n-1 \mid 1)-p(0 \mid 1) \cdot p(n-1 \mid 0)} \cdot \sum_{l=1}^{n} p(l-1 \mid 1) \cdot q(l) .
\end{aligned}
$$

Canceling yields,

$$
\begin{aligned}
& =\frac{p(0 \mid 0)}{p(0 \mid 1)} \cdot \sum_{l=1}^{n} p(l-1 \mid 1) \cdot q(l)-\frac{p(0 \mid 0)}{p(0 \mid 1)} \cdot \sum_{l=1}^{n} p(l-1 \mid 1) \cdot q(l) \\
& =0
\end{aligned}
$$


Therefore (IC0) is satisfied. Finally we have to show that (AB) and (EF) hold. Using Lemma 2 we can write $(\mathrm{AB})$ as:

$$
r \cdot \sum_{k=0}^{n} p(k) \cdot q(k) \geqslant g(1) \cdot \sum_{l=1}^{n} p(l-1 \mid 1) \cdot x_{l 1}+g(0) \cdot \sum_{l=0}^{n-1} p(l \mid 0) \cdot x_{l 0} .
$$

From (A1) the second term on the right hand side vanishes. Furthermore, since (VP1) holds with equality we can rewrite the first term on the right hand side. This yields: $r \cdot \sum_{k=0}^{n} p(k) \cdot q(k) \geqslant g(1) \cdot \sum_{l=1}^{n} p(l-1 \mid 1) \cdot q(l)$. Using again Lemma 2 the inequality simplifies, yielding: $\sum_{k=0}^{n} p(k) \cdot q(k)$. $[r-(k / n)] \geqslant 0$. For $k \leqslant r \cdot n$ the term in square brackets is non-negative and hence we have $(\mathrm{EF})$.

Q.E.D.

Proof of Lemma 3. Observe that the problem has a recursive structure and we can solve it in two steps: In the first one, which we denote problem $A$, we take $\left(q(l), x_{l 1}\right)_{l=0}^{n}$ as given and choose $\left(x_{l 0}\right)_{l=0}^{n-1}$ to minimize expected transfers to type 0 agents subject to (IC0) and (LL). Because of (LL), the (IC0) constraint will be binding, and we can express the optimal transfers to type 0 agents, $x_{l 0}$, as a function of $\left(q(l), x_{l 1}\right)_{l=0}^{n}$. In the second step, problem $B$, we choose $\left(x_{l 1}\right)_{l=1}^{n}$ to minimize expected transfers to agents of type 0 and type 1 , subject to (VP1) and (LL). (VP1) will be binding, and we can express $\left(x_{l 1}\right)_{l=1}^{n}$ as a function of $(q(l))_{l=0}^{n}$. Substituting in the optimal transfers $\left(x_{l 0}\right)_{l=0}^{n-1}$ yields the required result. Problem $\mathrm{A}$ is given by:

$$
\begin{aligned}
\min _{x_{l 0} \geqslant 0} & \sum_{l=0}^{n-1}(n-l) \cdot p(l) \cdot x_{l 0} \quad \text { subject to } \sum_{l=0}^{n-1} p(l \mid 0) \cdot x_{l 0} \\
& \geqslant \sum_{l=1}^{n} p(l-1 \mid 0) \cdot x_{l 1} .
\end{aligned}
$$

Lemma 2 shows that $p(l \mid 0) /((n-l) \cdot p(l))=(1 /(n \cdot g(0))$ and hence the objective function is equal to the left hand side of the constraint times $n \cdot g(0)$. The minimizing expenditures to type zero agents are therefore equal to $n \cdot g(0) \cdot \sum_{l=1}^{n} p(l-1 \mid 0) \cdot x_{l 1}$ and the optimal transfers to type zero agents is any combination of $x_{10}$ 's such that the constraint holds with equality. We choose arbitrarily $x_{00}^{*}=1 / p(0 \mid 0) \cdot \sum_{l=1}^{n} p(l-1 \mid 0) \cdot x_{l 1}$ and $x_{l 0}^{*}=0 \forall l>0$. Problem B is given by

$$
\begin{aligned}
& \min _{x_{l} \geqslant 0} \sum_{l=1}^{n}[l \cdot p(l)+p(l-1 \mid 0) \cdot n \cdot g(0)] \cdot x_{l 1} \\
& \quad \text { subject to } \sum_{l=1}^{n} p(l-1 \mid 1) \cdot x_{l 1} \geqslant \sum_{l=1}^{n} p(l-1 \mid 1) \cdot q(l) .
\end{aligned}
$$


Observe that the objective function and the left hand side of the constraint are both weighted sums of $x_{l 1}$, but the weights differ. The ratio of the weight in the objective function to the weight in the constraint is given by $(l \cdot p(l)+p(l-1 \mid 0) \cdot n \cdot g(0)) / p(l-1 \mid 1)$. Since Lemma 2 shows that $(l \cdot p(l)) / p(l-1 \mid 1)=n \cdot g(1)$, this ratio equals to $n \cdot[g(1)+g(0)$. $p(l-1 \mid 0) / p(l-1 \mid 1)]$. To solve problem $\mathrm{B}$ we have to find an $l^{*}$ that minimizes $p(l-1 \mid 0) / p(l-1 \mid 1)$, i.e., $l^{*} \in \operatorname{argmin} p(l-1 \mid 0) / p(l-1 \mid 1)$. Set $x_{l^{*} 1}=1 / p\left(l^{*}-1 \mid 1\right) \cdot \sum_{l=1}^{n} p(l-1 \mid 1) \cdot q(l)$ and $x_{l 1}=0$ for $l \neq l^{*}$. Next we show that $l^{*}=n$. Define $\phi(s)=s^{n-1}(1-s) f(s)$ and $\xi(s, l)=s^{l}(1-s)^{n-l} f(s)$ with $l<n-1$. Let $d(s, l)=(s /(1-s))^{n-l-1}\left(\int_{S} \xi(y, l) d y\right) /\left(\int_{S} \phi(y) d y\right)$ and observe that $d(s, l)$ is strict monotone increasing in $s$, for any $l<n-1$. Next observe that the ratio $(\phi(s) / \xi(s, l))\left(\int_{S} \xi(y, l) d y / \int_{S} \phi(y) d y\right)$ equals $d(s, l)$. So $\phi(s) / \int_{S} \phi(y) d y=d(s, l)\left(\xi(s, l) / \int_{S} \xi(y, l) d y\right)$.

Notice, that

$$
\int_{S} \frac{\phi(s)}{\int_{S} \phi(y) d y} d s=1=\int_{S} \frac{\xi(s, l)}{\int_{S} \xi(y, l) d y} d s \quad \text { for } \quad \text { all } \quad l<n-1,
$$

which can be rewritten as $\int_{S}[d(s, l)-1]\left(\xi(s, l) / \int_{S} \xi(y, l) d y\right) d s=0$. Since $d(s, l)$ is strict monotone increasing in $s$ and $\xi(s, l) / \int_{s} \xi(y, l) d y>0$, this implies that there exists an $a \in(0,1)$ such that $d(s, l)-1<0$ for $s<a$ and $d(s, l)-1>0$ for $s>a$. Now $s /(1-s)$ strict monotone increasing in $s$ implies that the following inequalities hold:

$$
\begin{aligned}
& \int_{S} \frac{s}{1-s}[d(s, l)-1] \frac{\xi(s, l)}{\int_{S} \xi(y, l) d y} \\
& \quad=\int_{0}^{a} \frac{s}{1-s}[d(s, l)-1] \frac{\xi(s, l)}{\int_{S} \xi(y, l) d y} d s+\int_{a}^{1} \frac{s}{1-s}[d(s, l)-1] \frac{\xi(s, l)}{\int_{S} \xi(y, l) d y} d s \\
& \quad>\int_{0}^{a} \frac{a}{1-a}[d(s, l)-1] \frac{\xi(s, l)}{\int_{S} \xi(y, l) d y} d s+\int_{a}^{1} \frac{a}{1-a}[d(s, l)-1] \frac{\xi(s, l)}{\int_{S} \xi(y, l) d y} d s \\
& \quad=\frac{a}{1-a} \int_{0}^{1}[d(s, l)-1] \frac{\xi(s, l)}{\int_{S} \xi(y, l) d y} d s \\
& \quad=0
\end{aligned}
$$

The first equality follows from breaking up the range of integration. The second inequality is strict. To see this observe that $\int_{0}^{a}[s /(1-s)-a /(1-a)]$ $[d(s, l)-1]\left(\xi(s, l) / \int_{s} \xi(y, l) d y\right) d s>0$ since $s /(1-s)<a /(1-a)$ for $s<a$ and $d(s, l)-1<0$ for $s<a$. Similarly, $\int_{a}^{1}[s /(1-s)-a /(1-a)][d(s, l)-1]$ $\left(\xi(s, l) / \int_{S} \xi(y, l) d y\right) d s>0$ since $s /(1-s)>a /(1-a)$ for $s>a$ and 
$d(s, l)-1>0$ for $s>a$. The third equality is obtained by rearranging and the last equality follows sinc $\int_{S}[d(s, l)-1]\left(\xi(s, l) / \int_{S} \xi(y, l) d y\right) d s=0$. Using the definition of $d(s, l)$ we can rewrite this as,

$$
\int_{S} \frac{s}{1-s} \frac{\phi(s)}{\int \phi(y) d y} d s>\int_{S} \frac{s}{1-s} \frac{\xi(s, l)}{\int \xi(y, l) d y} d s,
$$

which can be rewritten as

$$
\begin{aligned}
\frac{p(n-1 \mid 0)}{p(n-1 \mid 1)} & =\frac{\int \phi(s) d s}{\int \frac{s}{1-s} \phi(s) d s} \cdot \frac{g(1)}{g(0)}<\frac{\int \xi(s, l) d s}{\int \frac{s}{1-s} \xi(s, l) d s} \frac{g(1)}{g(0)} \\
& =\frac{p(l \mid 0)}{p(l \mid 1)} \quad \forall l<n-1,
\end{aligned}
$$

and thus the ratio $p(l-1 \mid 0) / p(l-1 \mid 1)$ is minimized at $l^{*}=n$. The optimal transfer payments are therefore $x_{n 1}^{*}=(1 / p(n-1 \mid 1)) \sum_{l=1}^{n} p(l-1 \mid 1) q(l)$, $x_{k 1}^{*}=0 \forall k<n$ and $x_{00}^{*}=(p(n-1 \mid 0) / p(n-1 \mid 1) p(0 \mid 0)) \sum_{l=1}^{n} p(l-1 \mid 1) q(l)$, $x_{k 0}^{*}=0 \forall k>0$. Finally the minimal expenditures in per capita terms are given by $p(0) x_{00}^{*}+(n / n) p(n) x_{n 1}^{*}$. Using the optimal transfers yields the stated result.

Q.E.D.

Proof of Theorem 6. We construct a mechanism that has a positive probability of provision and satisfies (IC), (VP), (PB), and (LL). The mechanism, which we call mechanism $(*)$, is characterized in terms of two variables $\delta, \varepsilon$ with $\varepsilon<(r / 2)$. The probability of provision is given by

$$
q(k)= \begin{cases}1 & \text { if } \frac{k}{n} \in[0, \varepsilon] \quad \text { or } \quad \frac{k}{n} \in\left[\frac{r}{2}, \frac{r}{2}+\delta\right] ; \\ 0 & \text { otherwise. }\end{cases}
$$

The transfers to agents of type 1 are given by

$$
x_{1}(k)= \begin{cases}\frac{r n}{k} & \text { if } \frac{k}{n} \in\left[\frac{r}{2}, \frac{r}{2}+\delta\right] ; \\ 0 & \text { otherwise; }\end{cases}
$$

and to agents of type 0 the transfer payments are given by

$$
x_{0}(k)= \begin{cases}\frac{r n}{n-k} & \text { if } \frac{k}{n} \in[0, \varepsilon] \\ 0 & \text { otherwise. }\end{cases}
$$


By construction this mechanism satisfies (PB) since the payments are always equal to the revenues. (LL) and (VP0) are satisfied since the payments are non-negative. We show that for $\delta, \varepsilon$ sufficiently small there exists an $N$ such that for all $n>N$ the constraints (VP1), (IC0), and (IC1) are satisfied. We will establish this via two lemmas. In the first we show that, for any $\delta, \varepsilon>0$ satisfying three conditions (A.5), (A.6), and (A.7), there exists an $N$ such that for $n>N$ the constraints are satisfied. In the second lemma we show that there exist $\delta, \varepsilon$ positive (but small) satisfying these three conditions: (A.5), (A.6), and (A.7). Since the probability density function $f$ has full support by Assumption (i), and the mechanism (*) provides the project when $k / n$ falls into the regions $[0, \varepsilon]$ or $[r / 2, r / 2+\delta]$, this completes the proof of the theorem. Before proceeding we give a notion of the limiting distribution of the variable fraction of type 1 agents. This notion will be used to give a bound for the probability of provision for large $n$ in the following lemmas.

Let $y$ denote the fraction of type 1 agents and let $F(y)$ denote $\int_{0}^{y} f(s) d s$. As $n$ goes to infinity the probability of the event that at most a fraction $y$ of all agents are of type 1, converges to the probability of the event that a state $s \leqslant y$ occurred. Let $p_{n}(k)$ be the probability that there are $k$ type ones if the number of agents equals $n$. Formally, for any $y \in[0,1]$, we have $\sum_{k=0}^{y \cdot n} p_{n}(k) \rightarrow \int_{0}^{y} f(s) d s$. (Hald [4] discusses the limiting distribution.) Since $F(\cdot)$ is the limiting distribution, for any $\gamma>0$ and for any fixed $x \leqslant y$, there exists an $N$ such that for all $n>N, F(y)-F(x)-\gamma<\sum_{k=x n}^{y n} p_{n}(k)<$ $F(y)-F(x)+\gamma$. (Here it is understood that the variable $k$ takes on only integer values even if $x n$ or $y n$ is not an integer.) So for given $\gamma, x, y$ this gives an approximation for the probability $\sum_{k=x n}^{y n} p_{n}(k)$ that holds for $n>N$.

Lemma 6. Consider mechanism (*). For $\varepsilon, \delta>0$ satisfying the following three conditions, there exists an $N$ such that for all $n>N$ the constraints (VP1), (IC0), and (IC1) are satisfied:

$$
\begin{gathered}
{\left[\frac{r}{2}-\delta\right]\left[F\left(\frac{r}{2}+\delta\right)-F\left(\frac{r}{2}\right)-F(\varepsilon) \varepsilon\right] \geqslant \varepsilon[F(\varepsilon)+F(\varepsilon) \varepsilon],} \\
F(\varepsilon)[1-\varepsilon] \geqslant \frac{2-r}{r}\left[F\left(\frac{r}{2}+\delta\right)-F\left(\frac{r}{2}\right)+F(\varepsilon) \varepsilon\right], \\
{\left[F\left(\frac{r}{2}+\delta\right)-F\left(\frac{r}{2}\right)-F(\varepsilon) \varepsilon\right] \geqslant \frac{\varepsilon}{1-\varepsilon}[F(\varepsilon)+\varepsilon F(\varepsilon)] .}
\end{gathered}
$$


Proof. Pick $\delta, \varepsilon>0$. We establish that under condition (A.5) there exists an $N$ such that for $n>N$ the (VP1) constraint is satisfied. Similarly we establish that under condition (A.6) there exists an $N$ such that for $n>N$ the (IC0) constraint is satisfied and that under condition (A.7) there exists an $N$ such that for $n>N$ the (IC1) constraint is satisfied. This implies that all constraints are satisfied if (A.5), (A.6), and (A.7) hold and $N$ is the largest of these three $N$ 's.

We begin with (VP1). The transfer payments $x_{1}(k)$ and the probability of provision $q(k)$ determine (VP1). It is given by

$$
\sum_{k=(r / 2) n}^{((r / 2)+\delta) n} \frac{r n}{k} p(k-1 \mid 1) \geqslant \sum_{k=1}^{\varepsilon n} p(k-1 \mid 1)+\sum_{k=(r / 2) n}^{((r / 2)+\delta) n} p(k-1 \mid 1) .
$$

After using $p(k-1 \mid 1)=k /(n g(1)) p_{n}(k)$ from Lemma 2, canceling $g(1)$ on both sides and bringing the second sum on the right hand side to the left hand side, the (VP1) constraint is given by

$$
\sum_{k=(r / 2) n}^{((r / 2)+\delta) n}\left(r-\frac{k}{n}\right) p_{n}(k) \geqslant \sum_{k=1}^{\varepsilon n} \frac{k}{n} p_{n}(k) .
$$

We can approximate the sums in this equation using the above limiting distribution for $p_{n}$. Specifically we show that there exists an $N$ such that (VP1) is satisfied for $n>N$ if (A.5) is satisfied. To see this, consider first the left hand side in (A.8). Observe that $\sum_{k=(r / 2) n}^{((r / 2)+\delta) n}(r-(k / n)) p_{n}(k) \geqslant$ $[(r / 2)-\delta] \sum_{k=(r / 2) n}^{((r / 2)+\delta) n} p_{n}(k) \geqslant[(r / 2)-\delta][F((r / 2)+\delta)-F((r / 2))-F(\varepsilon) \varepsilon]$ for large $n$. The first inequality uses that $r-(k / n) \geqslant(r / 2)-\delta$ for $k \leqslant((r / 2)+\delta) n$. The second inequality follows from the fact that for any $\gamma>0$ there exists an $N$ such that for all $n>N, \sum_{k=(r / 2) n}^{((r / 2)+\delta) n} p_{n}(k) \geqslant$ $[F((r / 2)+\delta)-F(r / 2)-\gamma]$. Using $\gamma=F(\varepsilon) \varepsilon$ yields the second inequality. So the left hand side in (A.8) is greater than the left hand side in (A.5). Consider next the right hand side in (A.8). The following inequalities are satisfied for large $n . \sum_{k=1}^{\varepsilon n}(k / n) p_{n}(k) \leqslant \varepsilon \sum_{k=1}^{\varepsilon n} p_{n}(k) \leqslant \varepsilon[F(\varepsilon)+F(\varepsilon) \varepsilon]$. To see the first inequality observe that $(k / n) \leqslant \varepsilon$ for $k \leqslant \varepsilon n$. To see the second inequality observe that for any $\gamma>0$ there exists an $N$ such that for $n>N$, $\sum_{k=1}^{\varepsilon n} p_{n}(k) \leqslant F(\varepsilon)-F(0)+\gamma$. Using $\gamma=F(\varepsilon) \varepsilon$ and $F(0)=0$ yields the second inequality. The right hand side in (A.5) is greater than the right hand side in (A.8). So, for any $\delta$, $\varepsilon$, satisfying (A.5), there exists an $N$ such that for all $n>N$ the constraint (VP1) is satisfied.

Next consider (IC0). The transfer payments $x_{0}(k), x_{1}(k)$ and the probability of provision $q(k)$ determine (IC0). It is given by,

$$
\sum_{k=0}^{\varepsilon n} \frac{r n}{n-k} p(k \mid 0) \geqslant \sum_{k=(r / 2) n}^{((r / 2)+\delta) n} \frac{r n}{k} p(k-1 \mid 0) .
$$


After using $p(k \mid 0)=((n-k) / n g(0)) p_{n}(k)$ from Lemma 2 and canceling $r / g(0)$, the (IC0) constraint can be written as

$$
\sum_{k=0}^{\varepsilon n} p_{n}(k) \geqslant \sum_{k=(r / 2) n}^{((r / 2)+\delta) n} \frac{n-k+1}{k} p_{n}(k-1) .
$$

We again approximate the sums in Eq. (A.9) using the above limiting distribution of $p_{n}$ and this yields (A.6). Consider first the left hand side in (A.9). For any $\gamma>0$ there exists an $N$ such that for $n>N$ the sum $\sum_{k=0}^{\varepsilon n} p_{n}(k) \geqslant F(\varepsilon)-\gamma$. Using $\gamma=F(\varepsilon) \varepsilon$ implies that the left hand side in (A.9) is greater than the left hand side in (A.6). To see that the right hand side in (A.6) is greater than the right hand side in (A.9) consider the following inequalities,

$$
\begin{aligned}
& \sum_{k=(r / 2) n}^{((r / 2)+\delta) n} \frac{n-k+1}{k} p_{n}(k-1) \\
& \leqslant\left[\frac{2-r}{r}+\frac{2}{r n}\right] \sum_{k=(r / 2) n}^{((r / 2)+\delta) n} p_{n}(k-1) \\
& \quad \leqslant\left[\frac{2-r}{r}+\frac{2}{r n}\right]\left[\sum_{k=(r / 2) n}^{((r / 2)+\delta) n} p_{n}(k)+p_{n}\left(\frac{r}{2} n-1\right)\right] \\
& \quad \leqslant \frac{2-r}{r} \sum_{k=(r / 2) n}^{((r / 2)+\delta) n} p_{n}(k)+\frac{2}{r n} \cdot 1+p_{n}\left(\frac{r}{2} n-1\right) \cdot \frac{2-r}{r} \\
& \quad \leqslant \frac{2-r}{r}\left[F\left(\frac{r}{2}+\delta\right)-F\left(\frac{r}{2}\right)+\gamma_{1}\right]+\gamma_{2} .
\end{aligned}
$$

The first inequality uses that $(n-k+1) / k$ is monotone decreasing in $k$, and thus $(n-k+1) / k \leqslant(2-r) / r+2 /(r n)$ for $k \geqslant(r / 2) n$. The second inequality is obtained by augmenting the sum on the right hand side by $p_{n}(((r / 2)+\delta) n)$. The third inequality uses that the second term in square brackets is a probability and thus less than 1 . The last inequality uses two statements. The first is the approximation that for any $\gamma_{1}>0$ there exists an $N$ such that for $n>N, \sum_{k=(r / 2) n}^{((r / 2)+\delta) n} p_{n}(k) \leqslant F((r / 2)+\delta)-F((r / 2))+\gamma_{1}$. The second is that, $2 /(r n)$ and $p_{n}((r / 2) n-1) \cdot(2-r) / 2$, vanish as $n$ increases. To see that $p_{n}(x n) \rightarrow 0$ as $n \rightarrow \infty$ we can use the limiting distribution. For any $\gamma>0$ there exists $N$ such that for all $n>N, p_{n}(x n)=\sum_{k=x n}^{x n} p_{n}(k) \leqslant$ $F(x)-F(x)+\gamma=\gamma$. So for any $\gamma_{2}>0$ there exists an $N$ such that for $n>N, 2 /(r n)+p_{n}((r / 2) n-1)((2-r) / r)<\gamma_{2}$. Combining these two statements yields the last inequality. We can set $\gamma_{1}, \gamma_{2}$ such that $((2-r) / r) \gamma_{1}+\gamma_{2}=$ $((2-r) / r) F(\varepsilon) \varepsilon$ and this yields the right hand side in (A.6). Observe that the right hand side is at least as large as the right hand side in (A.9). So, 
if (A.6) is satisfied then there exists an $N$ such that for all $n>N$ the constraint (IC0) is satisfied.

Finally we consider (IC1). The transfer payments $x_{1}(k), x_{0}(k)$ and the probability of provision $q(k)$ determine (IC1). It is given by,

$$
\begin{aligned}
& \sum_{k=(r / 2) n}^{((r / 2)+\delta) n} \frac{r n}{k} p(k-1 \mid 1)-\sum_{k=1}^{\varepsilon n} p(k-1 \mid 1)-\sum_{k=(r / 2) n}^{((r / 2)+\delta) n} p(k-1 \mid 1) \\
& \geqslant \sum_{k=1}^{\varepsilon n+1} \frac{r n}{n-k+1} p(k-1 \mid 1)-\sum_{k=1}^{\varepsilon n+1} p(k-1 \mid 1) \\
& \quad-\sum_{k=(r / 2) n+1}^{((r / 2)+\delta) n+1} p(k-1 \mid 1) .
\end{aligned}
$$

Canceling equal terms on both sides of the inequality, using $p(k-1 \mid 1)=k /(n g(1)) p_{n}(k)$ from Lemma 2 and dividing by $r / g(1)$, implies that the $(\mathrm{IC} 1)$ is satisfied if

$$
\sum_{k=(r / 2) n}^{((r / 2)+\delta) n} p_{n}(k) \geqslant \sum_{k=1}^{\varepsilon n+1} \frac{k}{n-k+1} p_{n}(k)+\frac{1}{2} p_{n}\left(\frac{r n}{2}\right) .
$$

Observe that the right hand side in (A.10) is larger than the right hand side in (IC1), since we dropped the terms $-p(\varepsilon n \mid 1)$ and $-p((r / 2)+\delta) n \mid 1)$. We next use the limiting distribution to approximate the sums and show that (A.7) implies (A.10). First consider the left hand side in (A.10). For $\gamma>0$ there exists an $N$ such that for $n>N$ the left hand side in (A.10) is greater than $[F((r / 2)+\delta)-F((r / 2))-\gamma]$. Using $\gamma=F(\varepsilon) \varepsilon$ yields that the left hand side in (A.10) is larger than the left hand side in (A.7) for $n>N$. Consider next the right hand side in (A.10). Observe that $k /(n-k+1)$ is monotone increasing in $k$. Thus the right hand side in (A.10) is less than or equal to $(\varepsilon /(1-\varepsilon+(1 / n))) \sum_{k=1}^{\varepsilon n} p_{n}(k)+(\varepsilon+(1 / n)) /(1-\varepsilon) p_{n}(\varepsilon n+1)+\frac{1}{2} p_{n}(r n / 2)$. Using the above limiting distribution implies that there exists an $N$ such that for $n>N,(\varepsilon /(1-\varepsilon+(1 / n))) \sum_{k=1}^{\varepsilon n} p_{n}(k) \leqslant(\varepsilon /(1-\varepsilon))\left[F(\varepsilon)+\gamma_{1}\right]$. Observe that $p_{n}(\varepsilon n+1) \rightarrow 0$ and $p_{n}(r n / 2) \rightarrow 0$, as $n \rightarrow \infty$. To see this, we can use the limiting distribution. For any $x \in[0,1]$ and $\gamma>0$ there exists an $N$ such the for all $n>N, p_{n}(x n)=\sum_{k=x n}^{x n} p_{n}(k) \leqslant F(x)-F(x)+\gamma=\gamma$. Using this implies that there exists an $N$ such that for all $n>N$, $((\varepsilon+(1 / n)) /(1-\varepsilon)) p_{n}(\varepsilon n+1)+\frac{1}{2} p_{n}(r n / 2)<(\varepsilon /(1-\varepsilon)) \gamma_{2}$. Let $\gamma_{1}=\gamma_{2}=\frac{1}{2} F(\varepsilon) \varepsilon$ yields the right hand side in (A.7). So, there exists an $N$ such that for $n>N$ the right hand side in (A.10) is smaller than the right hand side in (A.7). Combining the two statements yields that if (A.7) is satisfied, then there exists an $N$ such that for $n>N$ (IC1) is satisfied.

Q.E.D. 
Lemma 7. There exist $\delta$, $\varepsilon$ positive such that constraints (A.5), (A.6), and (A.7) are satisfied.

Proof. We first establish that condition (A.5) implies condition (A.7). To see this, observe that $(r / 2)-\delta$ is positive for $\delta$ small and we can divide inequality (A.5) by this expression. This transformation yields:

$$
\left[F\left(\frac{r}{2}+\delta\right)-F\left(\frac{r}{2}\right)-F(\varepsilon) \varepsilon\right] \geqslant \frac{1}{(r / 2)-\delta} \varepsilon[F(\varepsilon)+F(\varepsilon) \varepsilon] .
$$

Observe that the left hand sides in (A.11) and (A.7) are equal. The right hand side in (A.11) is larger than the right hand side in (A.7) if $(r / 2)-\delta<1-\varepsilon$. This is indeed satisfied for $\delta, \varepsilon$ small since $r<1$. So condition (A.5) implies condition (A.7) for $\delta, \varepsilon$ small.

Next we show that (A.5) and (A.6) are satisfied for $\delta, \varepsilon$ small. Bringing the last term on the left hand side in (A.11) to the right, the condition is given by:

$$
F\left(\frac{r}{2}+\delta\right)-F\left(\frac{r}{2}\right) \geqslant \frac{1}{(r / 2)-\delta} \varepsilon F(\varepsilon)\left[1+\varepsilon+\frac{r}{2}-\delta\right],
$$

Similarly bringing the last term on the right hand side of (A.6) to the left, multiplying by $r /(2-r)$, and rearranging yields:

$$
F(\varepsilon)\left[1-\varepsilon-\varepsilon \frac{2-r}{r}\right] \frac{r}{2-r} \geqslant F\left(\frac{r}{2}+\delta\right)-F\left(\frac{r}{2}\right) .
$$

Next choose $\delta$ such that (A.13) is satisfied with equality. Observe that if $\varepsilon$ is small, but positive, then $\delta(\varepsilon)$ is also positive, by strict monotonicity and continuity of $F$. We can substitute (A.13) with equality in inequality (A.12). Canceling $F(\varepsilon)$ on both sides yields:

$$
\left[1-\varepsilon-\varepsilon \frac{2-r}{r}\right] \frac{r}{2-r} \geqslant \frac{1}{(r / 2)-\delta(\varepsilon)} \varepsilon\left[1+\varepsilon+\frac{r}{2}-\delta(\varepsilon)\right] .
$$

As $\varepsilon$ goes to zero the right hand side in (A.14) goes to zero, but the left hand side is positive and bounded away from zero. So for $\varepsilon$ sufficiently small (A.14), and hence (A.5), is satisfied. By construction (A.12), and hence (A.6), are satisfied as well. This completes the proof.

Q.E.D. 


\section{REFERENCES}

1. R. H. Coase, The problem of social cost, J. Law Econ. 3 (1960), 1-44.

2. J. Cremer and R. McLean, Optimal selling strategies under uncertainty for a discriminating monopolist when demands are interdependent, Econometrica 53 (1985), 345-361.

3. J. Cremer and R. McLean, Full extraction of the surplus in Bayesian and dominant strategy auctions, Econometrica 56 (1988), 1247-1257.

4. A. Hald, The mixed binomial distribution and the posterior distribution of $p$ for a continuous prior distribution, J. Roy. Statist. Soc. Series B 30 (1968), 359-367.

5. G. Mailath and A. Postlewaite, Asymmetric information bargaining problems with many agents, Rev. Econ. Stud. 57 (1990), 351-367.

6. P. McAfee and P. Reny, Correlated information and mechanism design, Econometrica 60 (1992), 395-421.

7. R. Myerson, Optimal auction design, Math. Oper. Res. 6 (1981), 58-73.

8. R. Rob, Pollution claim settlements under private information, J. Econ. Theory 47 (1989), 307-333.

9. J. Robert, Continuity in auction design, J. Econ. Theory 55 (1991), 169-179. 